\title{
A MIMETIC DISCRETIZATION METHOD FOR LINEAR ELASTICITY
}

\author{
LOURENCO BeirÃo DA VEIGA ${ }^{1}$
}

\begin{abstract}
A Mimetic Discretization method for the linear elasticity problem in mixed weakly symmetric form is developed. The scheme is shown to converge linearly in the mesh size, independently of the incompressibility parameter $\lambda$, provided the discrete scalar product satisfies two given conditions. Finally, a family of algebraic scalar products which respect the above conditions is detailed.
\end{abstract}

Mathematics Subject Classification. 65N30, 65N12, 74B05.

Received October 31, 2008. Revised July 3rd, 2009.

Published online January 27, 2010.

\section{INTRODUCTION}

The main characteristic of the Mimetic Discretization method (or Mimetic Finite Difference method, MFD), when compared to a more standard finite element approach, is that the basis functions related to the discrete degrees of freedom are not explicitly defined. As a consequence, the differential operators and the other quantities appearing in the problem are approximated by discrete counterparts that satisfy finite dimensional analogs of fundamental properties. This approach makes it possible to mimic intrinsic properties of the differential problem under study. Moreover, a great flexibility in the mesh definition is achieved. In particular, general polyhedral (or polygonal in two dimensions) meshes, even with non convex and non matching elements, are admissible.

Although the MFD method has been applied to a variety of problems, such as electromagnetics and gas dynamics, the main benchmark of development and application has been the diffusion problem, see for example [11,12,24-29]. Lately, in a series of papers [16-19], the authors has succeeded in developing a general convergence theory that covers a very large range of possible meshes. Furthermore, in the same papers an effective algebraic method for the construction of MFD methods for the diffusion problem is provided. In [8,9,23] a higher order version MFD schemed is developed, in [6,7] an a posteriori error estimator is proposed and analyzed, while in [20] a post-processing technique is investigated.

In the present contribution, we aim at developing an MFD method for the elasticity problem. We follow the mixed, or Hellinger-Reissner, formulation, which adopts stresses and displacements as unknowns. Such formulation holds a strong similarity with the diffusion problem. Indeed, up to a substitution of vectors with tensors and scalars with vectors, the structure of the two problems is very similar. On the other hand, there are

Keywords and phrases. Mimetic finite difference methods, linear elasticity, finite element methods, mixed formulation.

1 Dipartimento di Matematica, Università di Milano, Via Saldini 50, 20133 Milano, Italy. lourenco.beirao@unimi.it 
a pair of additional difficulties that make the numerical discretization and analysis of the elasticity problem more involved. The first one is the symmetry of the stress tensor, which in most methods is enforced through the variational equations rather than directly in the discrete space. The second one is the lack of uniform coercivity of the main bilinear form of the problem, which arises when the very important case of almost incompressible materials is considered. After the pioneering works [2,22], a range of stable and converging finite elements for the elasticity problem in mixed form has been developed in [3-5,15].

In this paper we extend the ideas of $[4,5,15]$ in order to construct an MFD method that is uniformly stable and convergent. The main difficulty lays in the fact that everything must be defined through the degrees of freedom, since in MFD schemes the basis functions are unknown. In particular, (1) we need to derive new discrete operators for anti-symmetry and trace that respect given properties, and (2) to develop a scalar product that mimics properly the non uniform coercivity of the inverse elastic moduli.

The contribution is organized as follows. In Section 2 we introduce the linear elasticity problem in mixed form with weakly imposed symmetry, and describe the proposed MFD method. Afterwards, in Section 3 we prove the stability and convergence of the method assuming certain properties of the involved discrete scalar product. In particular, we prove that the new MFD method is uniformly converging in the incompressibility parameter $\lambda$ with order $O(h)$, where $h$ is the maximum diameter of the mesh elements. Finally, in Section 4 we discuss the construction of a family of scalar products that are proved to satisfy the properties required in Section 3.

For the sake of presentation, in this contribution we consider the three dimensional case. The (simpler) two dimensional case can be easily derived by adapting the same arguments.

\section{Presentation of the Method}

In this section we present the linear elasticity deformation problem and the proposed Mimetic Finite Difference method. Throughout the paper, $L^{p}(\omega)$ denotes the standard space of $p$-Lebesgue integrable functions over the open domain $\omega$, with the usual extension in the case $p=\infty$. The symbol $H^{k}(\omega), k \in \mathbb{N}$, indicates the standard Sobolev space of order $k$ and while $H_{0}^{1}(\omega)$ denotes the space of functions in $H^{1}(\omega)$ vanishing on the boundary $\partial \omega$. Finally, $H_{\text {div }}(\Omega)$ represents the tensor fields in $\left[L^{2}(\Omega)\right]^{3 \times 3}$ whose divergence is in $\left[L^{2}(\Omega)\right]^{3}$.

\subsection{The linear elasticity problem}

Let $\Omega \in \mathbb{R}^{3}$ be a polyhedral domain occupied by a linear elastic body, which is blocked on part of the boundary $\Gamma^{\prime} \subset \partial \Omega$ and free on the remaining part $\Gamma$. Both $\Gamma$ and $\Gamma^{\prime}$ are assumed to be a finite union of connected components, with meas $\left(\Gamma^{\prime}\right)>0$ in order to eliminate the rigid body motions. The body is subjected to a load $\mathbf{f} \in L^{2}(\Omega)$. Then, following the classical theory of linear elasticity [21], the deformation of $\Omega$ is governed by the equations

$$
\begin{cases}\boldsymbol{\sigma}=\mathbb{C} \varepsilon(\mathbf{u}):=2 \mu \varepsilon(\mathbf{u})+\lambda \operatorname{tr}(\varepsilon(\mathbf{u})) \mathbb{I} & \text { in } \Omega \\ \operatorname{div} \boldsymbol{\sigma}=\mathbf{f} & \text { in } \Omega \\ \mathbf{u}=\mathbf{0} \quad \text { on } \Gamma^{\prime}, \quad \boldsymbol{\sigma} \cdot \mathbf{n}=\mathbf{0} & \text { on } \Gamma\end{cases}
$$

where $\boldsymbol{\sigma}$ represents the stress tensor field, $\mathbf{u}$ the displacement vector field, $\mathbb{C}$ the tensor of elastic moduli with $\mu, \lambda \in L^{\infty}(\Omega)$ the positive Lamè parameters, II the second order identity tensor, $\mathbf{n}$ the outward unit normal on the boundary of $\Omega, \varepsilon$ the standard symmetric gradient operator and div, tr the standard divergence and trace operators.

In order to discretize problem (2.1) through a mimetic approach, we consider the mixed weakly symmetric formulation, which is equivalent to the one above; see for example [3] for an exhaustive derivation and references. 
The variational form reads

$$
\begin{cases}\text { Find }(\boldsymbol{\sigma}, \mathbf{u}, \mathbf{s}) \in H_{\mathbf{d i v}}^{\Gamma}(\Omega) \times\left[L^{2}(\Omega)\right]^{3} \times\left[L^{2}(\Omega)\right]^{3} \text { such that } & \\ \int_{\Omega} \mathbb{C}^{-1} \boldsymbol{\sigma}: \boldsymbol{\tau}+\int_{\Omega} \mathbf{u} \cdot \operatorname{div} \boldsymbol{\tau}+\int_{\Omega} \mathbf{s} \cdot \mathbf{a s}(\boldsymbol{\tau})=0 & \forall \boldsymbol{\tau} \in H_{\mathbf{d i v}}^{\Gamma}(\Omega) \\ \int_{\Omega} \operatorname{div} \boldsymbol{\sigma} \cdot \mathbf{v}=\int_{\Omega} \mathbf{f} \cdot \mathbf{v} & \forall \mathbf{v} \in\left[L^{2}(\Omega)\right]^{3} \\ \int_{\Omega} \operatorname{as}(\boldsymbol{\sigma}) \cdot \mathbf{q}=0 & \forall \mathbf{q} \in\left[L^{2}(\Omega)\right]^{3}\end{cases}
$$

where the double dot stands for the standard contraction operator between tensors, the single dot denotes the standard scalar product between vectors, $H_{\mathbf{d i v}}^{\Gamma}(\Omega)$ indicates the tensor fields in $H_{\mathbf{d i v}}(\Omega)$ with normal component vanishing on $\Gamma$, and the anti-symmetry operator as $: \mathbb{R}^{3 \times 3} \rightarrow \mathbb{R}^{3}$ is defined by

$$
\operatorname{as}(\boldsymbol{\tau})=\left(\begin{array}{l}
\boldsymbol{\tau}_{12}-\boldsymbol{\tau}_{21} \\
\boldsymbol{\tau}_{13}-\boldsymbol{\tau}_{31} \\
\boldsymbol{\tau}_{23}-\boldsymbol{\tau}_{32}
\end{array}\right) \quad \forall \boldsymbol{\tau} \in \mathbb{R}^{3 \times 3},
$$

where the subindices indicate the single components of the tensor $\tau$.

The bilinear form $\int_{\Omega} \mathbb{C}^{-1} \boldsymbol{\sigma}: \boldsymbol{\tau}$ is symmetric and $L^{2}$-positive definite since

$$
\mathbb{C}^{-1} \boldsymbol{\tau}=\frac{1}{2 \mu} \boldsymbol{\tau}-\frac{\lambda}{2 \mu(2 \mu+3 \lambda)} \operatorname{tr}(\boldsymbol{\tau}) \mathbb{I}
$$

for all $3 \times 3$ tensors $\boldsymbol{\tau}$. Note that in the limit case of an incompressible material, i.e. for $\lambda \rightarrow \infty$, the coercivity on the whole space is lost and holds only on the subspace of traceless tensors. Indeed, for all $\boldsymbol{\theta} \in \mathbb{R}^{3 \times 3}$ we have

$$
\mathbb{C}^{-1} \boldsymbol{\theta}: \boldsymbol{\theta}=\frac{1}{2 \mu}\|\operatorname{dev}(\boldsymbol{\theta})\|^{2}+\frac{1}{2 \mu+3 \lambda}|\operatorname{tr}(\boldsymbol{\theta})|^{2} \geq \frac{1}{2 \mu}\|\operatorname{dev} \boldsymbol{\theta}\|^{2}
$$

where dev stands for the deviatoric operator and $\|\cdot\|$ for the standard norm on tensors induced by the contraction scalar product. The well posedness and existence of a unique solution for problem (2.2) is discussed for instance in Theorem 2.1 in [3].

\subsection{Method presentation}

In this section we present the proposed discrete method. We start introducing a set of notations, assumptions and preliminary results.

\subsubsection{Mesh notation and assumptions}

For exposition's sake, we find it convenient to adopt the mesh notation and assumptions introduced in [16]. Let $\Omega_{h}$ be a conformal partition of $\Omega$ into non-overlapping polyhedral elements, which matches the boundary subdivision $\partial \Omega=\Gamma^{\prime} \cap \Gamma$. We indicate with $\Sigma_{h}$ the set of all the element faces. For every element $E$ we denote its 3-dimensional Lebesgue measure by $|E|$, its barycenter by $\mathbf{x}_{E}$, its diameter by $h_{E}$, and the number of its faces by $m_{E}$. The notation $\partial E$ may denote the boundary of the element $E$ or the union of the element faces depending on the context. Similarly, for every face $e$ of an element $E$ we denote its 2-dimensional Lebesgue measure by $|e|$, its diameter by $h_{e}$, its barycenter by $\mathbf{x}_{e}$, its outward unit normal vector by $\mathbf{n}_{E}^{e}$, the number of its edges by $m_{e}$, and the 1-dimensional measure of the edge $l$ by $|l|$. In addition, we associate to each face $e$ a unit normal vector $\mathbf{n}_{e}$, which is assumed fixed once and for all. The mesh $\Omega_{h}$ is sub-indexed by the mesh size parameter defined, as usual, by $h=\sup _{E} h_{E}$. 
Since this work follows the framework of references $[16,17]$, we consider the same mesh regularity assumptions detailed in these works. We list these assumptions below for the sake of the reader's convenience and for future reference in the paper.

(M1) There exist two positive integers $m_{f a}$ and $m_{e d}$ such that every element $E$ has $m_{E} \leq m_{f a}$ faces, and every face $e$ has $m_{e} \leq m_{e d}$ edges.

(M2) There exist three positive constants $c_{v}, c_{a}, c_{l}$ (for volume, area and length, respectively) such that $c_{v} h_{E}^{3} \leq|E|, c_{a} h_{E}^{2} \leq|e|$, and $c_{l} h_{E} \leq|l|$ for every element $E$ and for all faces $e$ and edges $l$ of $e$.

(M3) There exists a positive number $c_{\tau}$ such that each element $E$ is star-shaped with respect to all points of a ball of radius $c_{\tau} h_{E}$ and center at a point $M_{E} \in E$.

(M4) There exists a positive number $c_{\gamma}$ such that each face $e \in \partial E$ is star-shaped with respect to all points of a ball of radius $c_{\gamma} h_{E}$ and center at a point $M_{e} \in e$.

(M5) For every element $E$ and for every face $e \in \partial E$ there exists a pyramid contained in $E$ and having base coincident with $e$, height equal to $c_{\gamma} h_{E}$, and such that the projection of its vertex onto $e$ is $M_{e}$.

Finally, we assume for simplicity that the material constants $\mu$ and $\lambda$ are piecewise constant. This assumption can be interpreted as a data approximation. Moreover, we assume that it exists two positive constants $\mu_{*}$ and $\mu^{*}$ independent of $h$ such that

$$
\mu_{*} \leq \mu(x) \leq \mu^{*} \quad \forall \mathbf{x} \in \Omega
$$

Note that we do not make any additional assumption on $\lambda$, in order to include the important case of almost incompressible materials.

\subsubsection{Discrete spaces $Q_{h}$ and $X_{h}$}

Let us introduce the discrete spaces used for stresses, displacements and anti-symmetry Lagrange multipliers. Such finite dimensional spaces are represented by a set of degrees of freedom associated to elements or faces.

We introduce the finite dimensional space $Q_{h}$, whose degrees of freedom are a collection of discrete vectors in $\mathbb{R}^{3}$, associated to the elements $E$ of $\Omega_{h}$

$$
\mathbf{q}_{h} \in Q_{h} \Longrightarrow \mathbf{q}_{h}=\left\{\mathbf{q}_{E}\right\}_{E \in \Omega_{h}}, \mathbf{q}_{E} \in \mathbb{R}^{3}
$$

The dimension of $Q_{h}$ is therefore three times the number of elements, and each vector $\mathbf{q}_{h} \in Q_{h}$ is naturally associated to the piecewise constant vector field $\Omega \rightarrow \mathbb{R}^{3}$ that takes the indicated values in each element. With a little abuse of notation, we identify the elements of $Q_{h}$ also with the respective piecewise constant vector field.

Finally, we introduce the interpolation operator in $Q_{h}$, that is defined by

$$
\left(\mathbf{q}_{I}\right)_{E}=\frac{1}{|E|} \int_{E} \mathbf{q} \mathrm{d} \mathbf{x} \quad \forall E \in \Omega_{h},
$$

for any $\mathbf{q} \in\left[L^{1}(\Omega)\right]^{3}$, where integrals of vectors are interpreted component by component.

We introduce also the finite dimensional space $X_{h}$, whose degrees of freedom are a collection of vectors in $\mathbb{R}^{9}$, associated to the faces $e$ of $\Sigma_{h}$

$$
\boldsymbol{\tau}_{h} \in X_{h} \Longrightarrow \boldsymbol{\tau}_{h}=\left\{\left(\widehat{\boldsymbol{\tau}}_{e}, \widetilde{\boldsymbol{\tau}}_{e}^{1}, \widetilde{\boldsymbol{\tau}}_{e}^{2}\right)\right\}_{e \in \Omega_{h}}, \widehat{\boldsymbol{\tau}}_{e}, \widetilde{\boldsymbol{\tau}}_{e}^{1}, \widetilde{\boldsymbol{\tau}}_{e}^{2} \in \mathbb{R}^{3}
$$

The dimension of $X_{h}$ is therefore nine times the number of faces. Given any face $e \in \Sigma_{h}$, let $\boldsymbol{\xi}=\left(\xi_{1}, \xi_{2}\right) \in \mathbb{R}^{2}$ indicate the position vector of the face points with respect to a local coordinate system chosen on $e$ with the origin in the barycenter of $e$. Each local vector $\left(\widehat{\boldsymbol{\tau}}_{e}, \widetilde{\boldsymbol{\tau}}_{e}^{1}, \widetilde{\boldsymbol{\tau}}_{e}^{2}\right)$ is then associated to the linear vector field living on the face $e$ given by

$$
\boldsymbol{\tau}_{e}: e \rightarrow \mathbb{R}^{3}, \quad \boldsymbol{\tau}_{e}(\boldsymbol{\xi})=\widehat{\boldsymbol{\tau}}_{e}+\frac{1}{h_{e}} \widetilde{\boldsymbol{\tau}}_{e}^{1} \xi_{1}+\frac{1}{h_{e}} \widetilde{\boldsymbol{\tau}}_{e}^{2} \xi_{2}
$$

In the following the symbol $\boldsymbol{\tau}_{e}$ may denote both the vector $\left(\widehat{\boldsymbol{\tau}}_{e}, \widetilde{\boldsymbol{\tau}}_{e}^{1}, \widetilde{\boldsymbol{\tau}}_{e}^{2}\right)$ and the respective linear function (2.9). Thus, any element $\boldsymbol{\tau}_{h} \in X_{h}$ can also be written $\boldsymbol{\tau}_{h}=\left\{\boldsymbol{\tau}_{e}\right\}_{e \in \Sigma_{h}}$ and represent, for each face $e$, the normal 
component of a piecewise regular stress tensor field on $\Omega$. In this respect, we also use the notation

$$
\boldsymbol{\tau}_{E}^{e}=\left(\mathbf{n}_{E}^{e} \cdot \mathbf{n}_{e}\right) \boldsymbol{\tau}_{e} \quad \forall E \in \Omega_{h}, e \in \partial E,
$$

which represents the outward normal component of the virtual tensor field with respect to the element $E$.

For any tensor field $\boldsymbol{\tau} \in\left[L^{s}(\Omega)\right]^{3 \times 3}, s>2$, with $\operatorname{div} \boldsymbol{\tau} \in\left[L^{2}(\Omega)\right]^{3}$, we define the interpolant $\boldsymbol{\tau}_{I}$ in $X_{h}$ by

$$
\int_{e}\left(\boldsymbol{\tau}_{I}\right)_{e} \cdot \mathbf{p}^{1} \mathrm{~d} \boldsymbol{\xi}=\int_{e}\left(\boldsymbol{\tau} \mathbf{n}_{e}\right) \cdot \mathbf{p}^{1} \mathrm{~d} \boldsymbol{\xi} \quad \forall \mathbf{p}^{1} \in\left[\mathcal{P}_{1}(e)\right]^{3}, \forall e \in \Sigma_{h},
$$

where $\mathcal{P}_{1}(e)$ indicates the space of first order polynomials on $e$ and, here and in the following, a vector following a tensor represents the standard tensor-vector application

$$
(\boldsymbol{\theta} \mathbf{v}) \in \mathbb{R}^{n_{1}},(\boldsymbol{\theta} \mathbf{v})_{i}=\sum_{j=1}^{n_{2}} \boldsymbol{\theta}_{i j} \mathbf{v}_{j}, \quad i=1,2, \ldots, n_{1}, \boldsymbol{\theta} \in \mathbb{R}^{n_{1} \times n_{2}}, \mathbf{v} \in \mathbb{R}^{n_{2}}, n_{1}, n_{2} \in \mathbb{N} .
$$

It is easy to see that such interpolant is well defined and unique. In the paper there will be no confusion between the interpolation operators in $X_{h}$ and $Q_{h}$ since the first is applied to tensor fields and the second to vector fields.

Finally, the symbol $X_{h}^{\Gamma}$ denotes the subspace of the elements of $X_{h}$ that vanish on all the faces $e \in \Gamma$.

\subsubsection{Discrete operators on $X_{h}$}

We now define three linear discrete operators on the space $X_{h}$, which mimic respectively the standard operators of divergence, trace and anti-symmetry.

The discrete divergence operator $\operatorname{div}_{h}: X_{h} \rightarrow Q_{h}$ is defined by

$$
\left(\operatorname{div}_{h} \boldsymbol{\tau}_{h}\right)_{E}=\frac{1}{|E|} \sum_{e \in \partial E} \int_{e} \boldsymbol{\tau}_{E}^{e} \mathrm{~d} \boldsymbol{\xi} \quad \forall E \in \Omega_{h}
$$

which is consistent with the Gauss divergence theorem. Analogously as shown in [8,16], it is easy to check that the following commuting diagram property holds

$$
\operatorname{div}_{h}\left(\boldsymbol{\tau}_{I}\right)=(\operatorname{div} \boldsymbol{\tau})_{I} \quad \forall \boldsymbol{\tau} \in\left[L^{s}(\Omega)\right]^{3 \times 3} \cap H_{\operatorname{div}}(\Omega), s>2 .
$$

For each element $E$, we consider the vector-valued linear functions

$$
\boldsymbol{\varphi}_{E}=\left(\begin{array}{l}
x-x_{E} \\
y-y_{E} \\
z-z_{E}
\end{array}\right), \boldsymbol{\psi}_{E}^{1}=\left(\begin{array}{c}
y-y_{E} \\
x_{E}-x \\
0
\end{array}\right), \boldsymbol{\psi}_{E}^{2}=\left(\begin{array}{c}
z-z_{E} \\
0 \\
x_{E}-x
\end{array}\right), \boldsymbol{\psi}_{E}^{3}=\left(\begin{array}{c}
0 \\
z-z_{E} \\
y_{E}-y
\end{array}\right),
$$

where $\mathbf{x}=(x, y, z)$ represents the coordinates with respect to a global Cartesian system in $\Omega$ and $\mathbf{x}_{E}=$ $\left(x_{E}, y_{E}, z_{E}\right)$ indicates the barycenter of $E$. Note that $\nabla \varphi_{E}=\mathbb{I}$.

Using an integration by parts and observing that the integral of $\varphi_{E}$ on $E$ is null, it follows that

$$
\int_{E} \operatorname{tr}(\boldsymbol{\tau}) \mathrm{d} \mathbf{x}=\int_{E} \boldsymbol{\tau}: \boldsymbol{\nabla} \boldsymbol{\varphi}_{E} \mathrm{~d} \mathbf{x}=\sum_{e \in \partial E} \int_{e}\left(\boldsymbol{\tau} \mathbf{n}_{E}^{e}\right) \cdot \boldsymbol{\varphi}_{E} \mathrm{~d} \boldsymbol{\xi}
$$

for any tensor field $\boldsymbol{\tau} \in\left[L^{s}(E)\right]^{3 \times 3}, s>2$, whose divergence is constant.

Consistently, we define the discrete trace operator from $X_{h}$ into the space of $\Omega_{h}$-piecewise constant scalar functions as

$$
\operatorname{tr}_{h}\left(\boldsymbol{\tau}_{h}\right)_{E}=\frac{1}{|E|} \sum_{e \in \partial E} \int_{e} \boldsymbol{\tau}_{E}^{e} \cdot \boldsymbol{\varphi}_{E} \mathrm{~d} \boldsymbol{\xi} .
$$


From definition (2.11) and observing that $\varphi_{E}$ is linear, it follows that

$$
\sum_{e \in \partial E} \int_{e}\left(\boldsymbol{\tau} \mathbf{n}_{E}^{e}\right) \cdot \boldsymbol{\varphi}_{E} \mathrm{~d} \boldsymbol{\xi}=\sum_{e \in \partial E} \int_{e}\left(\boldsymbol{\tau}_{I}\right)_{E}^{e} \cdot \boldsymbol{\varphi}_{E} \mathrm{~d} \boldsymbol{\xi}
$$

for all sufficiently regular $\boldsymbol{\tau}$. By combining definition (2.16) and identities (2.15), (2.17) we get

$$
\operatorname{tr}_{h}\left(\boldsymbol{\tau}_{I}\right)_{E}=\frac{1}{|E|} \int_{E} \operatorname{tr}(\boldsymbol{\tau}) \mathrm{d} \mathbf{x} \quad \forall E \in \Omega_{h},
$$

for all $\tau \in\left[L^{s}(E)\right]^{3 \times 3}, s>2$, whose divergence is constant.

Recalling definition (2.3), we now observe that

$$
[\operatorname{as}(\boldsymbol{\theta})]_{i}=\boldsymbol{\theta}: \nabla \psi_{E}^{i}, \quad i=1,2,3, \forall \boldsymbol{\theta} \in \mathbb{R}^{3 \times 3},
$$

independently of $E$. Therefore, the same argument as in (2.15) gives

$$
\int_{E}[\operatorname{as}(\boldsymbol{\tau})]_{i} \mathrm{~d} \mathbf{x}=\sum_{e \in \partial E} \int_{e}\left(\boldsymbol{\tau} \mathbf{n}_{E}^{e}\right) \cdot \boldsymbol{\psi}_{E}^{i} \mathrm{~d} \boldsymbol{\xi}
$$

for all $\tau \in\left[L^{s}(E)\right]^{3 \times 3}, s>2$, whose divergence is constant. This fact leads to the following consistent definition of anti-symmetry operator from $X_{h}$ into $Q_{h}$

$$
\left[\operatorname{as}_{h}\left(\boldsymbol{\tau}_{h}\right)_{E}\right]_{i}=\frac{1}{|E|} \sum_{e \in \partial E} \int_{e} \boldsymbol{\tau}_{E}^{e} \cdot \boldsymbol{\psi}_{E}^{i} \mathrm{~d} \boldsymbol{\xi}, \quad i=1,2,3
$$

By applying the same argument used to show (2.18), we get

$$
\operatorname{as}_{h}\left(\boldsymbol{\tau}_{I}\right)_{E}=\frac{1}{|E|} \int_{E} \text { as }(\boldsymbol{\tau}) \mathrm{d} \mathbf{x} \quad \forall E \in \Omega_{h},
$$

for all $\tau \in\left[L^{s}(E)\right]^{3 \times 3}, s>2$, whose divergence is constant.

2.2.4. Scalar products, bilinear forms and norms

We equip $Q_{h}$ with the $L^{2}$ scalar product

$$
\left[\mathbf{q}_{h}, \mathbf{p}_{h}\right]_{Q_{h}}=\sum_{E \in \Omega_{h}}\left[\mathbf{q}_{h}, \mathbf{p}_{h}\right]_{E} \quad \text { with } \quad\left[\mathbf{q}_{h}, \mathbf{p}_{h}\right]_{E}=|E| \mathbf{q}_{E} \cdot \mathbf{p}_{E} \quad \forall \mathbf{q}_{h}, \mathbf{p}_{h} \in Q_{h},
$$

and indicate the induced global and local norms by $\|\cdot\|_{Q_{h}}$ and $\|\cdot\|_{E}$.

Regarding $X_{h}$, we first define the following norm

$$
\left\|\left|\boldsymbol{\tau}_{h}\left\|\left.\right|_{X_{h}} ^{2}=\sum_{E \in X_{h}}\right\| \boldsymbol{\tau}_{h} \|\right|_{E}^{2} \quad \text { with } \quad\right\| \boldsymbol{\tau}_{h}\left\|\left.\right|_{E} ^{2}=\sum_{e \in \partial E} h_{e}\right\| \boldsymbol{\tau}_{e} \|_{L^{2}(e)}^{2} \quad \forall \boldsymbol{\tau}_{h} \in X_{h} .
$$

We now need to define a scalar product on $X_{h}$ which mimics the natural form of the continuous problem $\left(\mathbb{C}^{-1} \cdot, \cdot\right)$, see $(2.4)$. We therefore assume the existence of a scalar product in $X_{h}$ with the properties that follow. In Section 4 we will show an example of construction of a scalar product that satisfies the involved conditions. Let therefore

$$
\left[\boldsymbol{\tau}_{h}, \boldsymbol{\delta}_{h}\right]_{X_{h}}=\sum_{E \in \Omega_{h}}\left[\boldsymbol{\tau}_{h}, \boldsymbol{\delta}_{h}\right]_{E} \quad \forall \boldsymbol{\tau}_{h}, \boldsymbol{\delta}_{h} \in X_{h}
$$


where the local forms are positive definite and depend only on the local values $\left\{\boldsymbol{\tau}_{E}^{e}\right\}_{e \in \partial E}$. Moreover, the forms $[\cdot, \cdot]_{E}$ are required to satisfy the two following conditions for all $E \in \Omega_{h}$.

(S1) There exist two positive constants $C_{*}, C^{*}$ independent of $h$ and $\lambda$ such that

$$
\left[\boldsymbol{\tau}_{h}, \boldsymbol{\delta}_{h}\right]_{E} \leq C^{*}||\left|\boldsymbol{\tau}_{h}\right|\left\|_{E}|| \boldsymbol{\delta}_{h} \mid\right\|_{E} \quad \forall \boldsymbol{\tau}_{h}, \boldsymbol{\delta}_{h} \in X_{h},
$$

and

$$
C_{*}\left|\left\|\boldsymbol{\tau}_{h}-\frac{1}{3} \operatorname{tr}_{h}\left(\boldsymbol{\tau}_{h}\right) \mathbb{I}_{I}\right\|\right|_{E}^{2} \leq\left[\boldsymbol{\tau}_{h}, \boldsymbol{\tau}_{h}\right]_{E} \quad \forall \boldsymbol{\tau}_{h} \in X_{h} \text { with } \operatorname{div}_{h} \boldsymbol{\tau}_{h}=0,
$$

where $\mathbb{I}_{I}$ is the interpolant in $X_{h}$ of the constant tensor $\mathbb{I}$.

(S2) For all $\boldsymbol{\tau}_{h} \in X_{h}$ there holds

$$
\left[\left(\mathbb{C} \nabla \mathbf{p}^{1}\right)_{I}, \boldsymbol{\tau}_{h}\right]_{E}+\int_{E} \mathbf{p}^{1} \cdot \operatorname{div}_{h} \boldsymbol{\tau}_{h} \mathrm{~d} \mathbf{x}=\sum_{e \in \partial E} \int_{e} \boldsymbol{\tau}_{E}^{e} \cdot \mathbf{p}^{1} \mathrm{~d} \boldsymbol{\xi}
$$

for all linear test functions $\mathbf{p}^{1} \in\left[\mathcal{P}_{1}(E)\right]^{3}$.

The first assumption represents the correct scaling of the form with respect to the size of the element, and a discrete type coercivity on the deviatoric part of the tensor, mimicking (2.5). The second assumption represents a consistency condition, stating that the scalar product respects the standard integration by parts when restricted to linear test functions.

We can now present the proposed method.

Method 2.1. Find $\left(\boldsymbol{\sigma}_{h}, \mathbf{u}_{h}, \mathbf{s}_{h}\right) \in X_{h}^{\Gamma} \times Q_{h} \times Q_{h}$ such that

$$
\begin{cases}{\left[\boldsymbol{\sigma}_{h}, \boldsymbol{\tau}_{h}\right]_{X_{h}}+\left[\mathbf{u}_{h}, \boldsymbol{d i v}_{h} \boldsymbol{\tau}_{h}\right]_{Q_{h}}+\left[\mathbf{s}_{h}, \mathbf{a s}_{h}\left(\boldsymbol{\tau}_{h}\right)\right]_{Q_{h}}=0} & \forall \boldsymbol{\tau}_{h} \in X_{h}^{\Gamma} \\ {\left[\operatorname{div}_{h} \boldsymbol{\sigma}_{h}, \mathbf{v}_{h}\right]_{Q_{h}}=\left[\mathbf{f}_{I}, \mathbf{v}_{h}\right]_{Q_{h}}} & \forall \mathbf{v}_{h} \in Q_{h} \\ {\left[\operatorname{as}_{h}\left(\boldsymbol{\sigma}_{h}\right), \mathbf{q}_{h}\right]_{Q_{h}}=0} & \forall \mathbf{q}_{h} \in Q_{h} .\end{cases}
$$

Note that the second two equations are equivalent to $\operatorname{div}_{h} \boldsymbol{\sigma}_{h}=\mathbf{f}_{I}$ and $\operatorname{as}_{h}\left(\boldsymbol{\sigma}_{h}\right)=\mathbf{0}$.

Remark 2.1. In analogy with [3,4], in the case meas $(\Gamma)=0$ the discrete stresses must be restricted to the subspace

$$
\left\{\boldsymbol{\tau}_{h} \in X_{h}\left|\sum_{E \in \Omega_{h}}\right| E \mid \operatorname{tr}_{h}\left(\boldsymbol{\tau}_{h}\right)_{E}=0\right\} .
$$

To ease presentation, we assume the more realistic case of meas $(\Gamma)>0$. The small modifications of the following proofs and statements in the case meas $(\Gamma)=0$ are rather standard and will not be considered here.

\section{Stability And CONVERGEnCE OF The Method}

In this section we prove the stability of the discrete Method 2.1 and its convergence properties. We use the symbols $\simeq, \lesssim, \gtrsim$ to represent equivalences and bounds that hold up to a constant independent of $h$ and $\lambda$.

We also use an auxiliary mesh whose construction is as follows. Given the mesh $\Omega_{h}$, we subdivide each face $e$ of $\Sigma_{h}$ in sub-triangles, connecting the vertices of each face $e$ to the point $M_{e}$ introduced in (M4). We call this set of triangles $\Xi_{e}$. Then, we subdivide each element $E$ into tetrahedrons, connecting the vertices of each triangle in $\Xi_{e}, e \in \partial E$, to the point $M_{E}$ introduced in (M3). This construction gives a conforming mesh of tetrahedrons for $\Omega$ which we call $\Omega_{h}^{\prime}$. In [6] it is proved that, under our mesh assumptions of Section 2.2.1, the family $\left\{\Omega_{h}^{\prime}\right\}_{h}$ is a shape-regular family of meshes.

Due to the shape regularity of $\left\{\Omega_{h}^{\prime}\right\}_{h}$, the stability of our method can be derived from the stability of a $B D M$ type tetrahedral finite element, as shown in the next section. Given the sub-triangulation $\Omega_{h}^{\prime}$ introduced above, let $V_{h}^{\Gamma}$ indicate the discrete tensor field given by three instances of the Brezzi-Douglas-Marini family of order 1, 
see [14], which satisfies the homogeneous boundary conditions for the normal component on $\Gamma$. Moreover, let $P_{h}$ represent the space of piecewise constant vector functions on the same triangulation. Then, the proof of the following result can be easily checked combining the proof of Theorem 4.6 in [15] with Example 7 in the same paper.

Proposition 3.1. It exists a positive constant $\beta^{\prime}$ independent of $h$ such that, for all $\mathbf{v}_{h}^{\prime}, \mathbf{q}_{h}^{\prime} \in P_{h}$, it exists a $\delta_{h}^{\prime} \in V_{h}^{\Gamma}$ satisfying

$$
\begin{aligned}
& \left(\mathbf{v}_{h}^{\prime}, \operatorname{div} \boldsymbol{\delta}_{h}^{\prime}\right)_{L^{2}(\Omega)}+\left(\mathbf{q}_{h}^{\prime}, \operatorname{as}\left(\boldsymbol{\delta}_{h}^{\prime}\right)\right)_{L^{2}(\Omega)} \geq \beta^{\prime}\left(\left\|\mathbf{v}_{h}^{\prime}\right\|_{L^{2}(\Omega)}+\left\|\mathbf{q}_{h}^{\prime}\right\|_{L^{2}(\Omega)}\right) \\
& \left\|\boldsymbol{\delta}_{h}^{\prime}\right\|_{L^{2}(\Omega)} \lesssim 1, \quad \operatorname{div} \boldsymbol{\delta}_{h}^{\prime}=\gamma^{\prime} \mathbf{v}_{h}^{\prime},
\end{aligned}
$$

where the scalar $\gamma^{\prime}=\left(\left\|\mathbf{v}_{h}^{\prime}\right\|_{L^{2}(\Omega)}+\left\|\mathbf{q}_{h}^{\prime}\right\|_{L^{2}(\Omega)}\right)^{-1}$. Furthermore, it exists a positive constant $\alpha^{\prime}$ independent of $h$ such that

$$
\left\|\operatorname{dev}\left(\boldsymbol{\delta}_{h}\right)\right\|_{L^{2}(\Omega)} \geq \alpha^{\prime}\left\|\boldsymbol{\delta}_{h}\right\|_{L^{2}(\Omega)} \quad \forall \boldsymbol{\delta}_{h} \in V_{h}^{\Gamma} \text { with } \operatorname{div} \boldsymbol{\delta}_{h}=\mathbf{0},
$$

where $\mathbf{d e v}$ stands for the operator extracting the deviatoric part of the tensor.

Note that, due to the classical theory in [13], the two conditions above ensure the uniform stability of the problem

$$
\begin{cases}\text { Find } \boldsymbol{\theta}_{h}^{\prime} \in V_{h}^{\Gamma}, \mathbf{v}_{h}^{\prime} \in P_{h} \text { such that } & \\ \left(\operatorname{dev}\left(\boldsymbol{\theta}_{h}^{\prime}\right), \operatorname{dev}\left(\boldsymbol{\delta}_{h}^{\prime}\right)\right)_{L^{2}(\Omega)}+\left(\mathbf{v}_{h}^{\prime}, \operatorname{div} \boldsymbol{\delta}_{h}^{\prime}\right)_{L^{2}(E)}=0 & \forall \boldsymbol{\delta}_{h}^{\prime} \in V_{h}^{\Gamma} \\ \left(\operatorname{div} \boldsymbol{\theta}_{h}^{\prime}, \mathbf{w}_{h}^{\prime}\right)_{L^{2}(\Omega)}=0 & \forall \mathbf{w}_{h}^{\prime} \in P_{h},\end{cases}
$$

where $V_{h}^{\Gamma}$ is equipped with the $H_{\text {div }}(\Omega)$ norm and the remaining discrete spaces are equipped with the $L^{2}(\Omega)$ norm.

\subsection{Uniform stability of the method}

We prove the stability of Method 2.1 on $X_{h}^{\Gamma} \times Q_{h} \times Q_{h}$, where the three spaces are equipped with the norms introduced in the previous section. Following the general theory of [13], this result stems directly from the discrete inf-sup condition, shown in Lemma 3.1 and the coercivity on the kernel condition, shown in Lemma 3.2.

Lemma 3.1. It exists a positive constant $\beta$ independent of $h$ such that, for all $\mathbf{v}_{h}, \mathbf{q}_{h} \in Q_{h}$, it exists $\boldsymbol{\tau}_{h} \in X_{h}$ satisfying

$$
\left[\mathbf{v}_{h}, \operatorname{div}_{h} \boldsymbol{\tau}_{h}\right]_{Q_{h}}+\left[\mathbf{q}_{h}, \operatorname{as}_{h}\left(\boldsymbol{\tau}_{h}\right)\right]_{Q_{h}} \geq \beta\left(\left\|\mathbf{v}_{h}\right\|_{Q_{h}}+\left\|\mathbf{q}_{h}\right\|_{Q_{h}}\right), \quad\left\|\boldsymbol{\tau}_{h}\right\| \|_{X_{h}} \lesssim 1 .
$$

Proof. Clearly, since $\Omega_{h}^{\prime}$ is obtained by a subdivision of $\Omega_{h}$, the functions $\mathbf{q}_{h}, \mathbf{v}_{h}$ belong to the space of the vector functions that are piecewise constant on $\Omega_{h}^{\prime}$. Let therefore $\boldsymbol{\tau}_{h}^{\prime}$ be the element of $V_{h}^{\Gamma}$ that satisfies condition (3.1) for the couple $\mathbf{q}_{h}, \mathbf{v}_{h}$ :

$$
\begin{aligned}
& \left(\mathbf{v}_{h}, \operatorname{div} \boldsymbol{\tau}_{h}^{\prime}\right)_{L^{2}(\Omega)}+\left(\mathbf{q}_{h}, \text { as }\left(\boldsymbol{\tau}_{h}^{\prime}\right)\right)_{L^{2}(\Omega)} \geq \beta^{\prime}\left(\left\|\mathbf{v}_{h}\right\|_{L^{2}(\Omega)}+\left\|\mathbf{q}_{h}\right\|_{L^{2}(\Omega)}\right), \\
& \left\|\boldsymbol{\tau}_{h}^{\prime}\right\|_{L^{2}(\Omega)} \lesssim 1, \quad \operatorname{div} \boldsymbol{\tau}_{h}^{\prime}=\gamma \mathbf{v}_{h},
\end{aligned}
$$

with $\gamma=\left(\left\|\mathbf{v}_{h}\right\|_{L^{2}(\Omega)}+\left\|\mathbf{q}_{h}\right\|_{L^{2}(\Omega)}\right)^{-1}$. Then, we define our candidate $\boldsymbol{\tau}_{h} \in X_{h}$ as the interpolant $\boldsymbol{\tau}_{h}=\left(\boldsymbol{\tau}_{h}^{\prime}\right)_{I}$.

Recalling the scalar product definition (2.21) and property (2.13), we have

$$
\left[\mathbf{v}_{h}, \operatorname{div}_{h} \boldsymbol{\tau}_{h}\right]_{Q_{h}}=\left(\mathbf{v}_{h}, \operatorname{div}_{h}\left(\boldsymbol{\tau}_{h}^{\prime}\right)_{I}\right)_{L^{2}(\Omega)}=\left(\mathbf{v}_{h}, \operatorname{div} \tau_{h}^{\prime}\right)_{L^{2}(\Omega)} .
$$


The last identity in (3.5) implies that the function $\operatorname{div} \boldsymbol{\tau}_{h}^{\prime}$ is $\Omega_{h}$-piecewise constant. Thus, we can apply (2.20) and, since also $\mathbf{q}_{h}$ is $\Omega_{h}$-piecewise constant, we obtain

$$
\left[\mathbf{q}_{h}, \mathbf{a s}_{h}\left(\boldsymbol{\tau}_{h}\right)\right]_{Q_{h}}=\left(\mathbf{q}_{h}, \mathbf{a s}_{h}\left(\left(\boldsymbol{\tau}_{h}^{\prime}\right)_{I}\right)\right)_{L^{2}(\Omega)}=\left(\mathbf{q}_{h}, \text { as }\left(\boldsymbol{\tau}_{h}^{\prime}\right)\right)_{L^{2}(\Omega)} .
$$

The first bound in (3.4) follows by combining (3.5), (3.6), (3.7) and using the definition of the norm in $Q_{h}$.

For all elements $E$ and faces $e \in \partial E,\left(\left(\boldsymbol{\tau}_{h}^{\prime}\right)_{I}\right)_{E}^{e}$ is by definition a $L^{2}(e)$ projection of $\boldsymbol{\tau}_{h}^{\prime} \mathbf{n}_{E}^{e}$ on the space of linear functions living on $e$. Therefore, from $(2.22)$ we get

$$
\left\|\left|\boldsymbol{\tau}_{h}\right|\right\|_{E}^{2}=\left\|||\left(\boldsymbol{\tau}_{h}^{\prime}\right)_{I} \mid\right\|_{E}^{2}=\sum_{e \in \partial E} h_{e}\left\|\left(\left(\boldsymbol{\tau}_{h}^{\prime}\right)_{I}\right)_{E}^{e}\right\|_{L^{2}(e)}^{2} \leq \sum_{e \in \partial E} h_{e}\left\|\boldsymbol{\tau}_{h}^{\prime} \mathbf{n}_{E}^{e}\right\|_{L^{2}(e)}^{2} .
$$

A standard scaling argument and (3.8) finally give

$$
\left\|\boldsymbol{\tau}_{h} \mid\right\|_{E}^{2} \lesssim\left\|\boldsymbol{\tau}_{h}^{\prime}\right\|_{L^{2}(E)}^{2}
$$

which, summing over all the elements $E \in \Omega_{h}$ and using (3.5), implies the second bound in (3.4).

Lemma 3.2. It exists a positive constant $\alpha$, independent of $h$ and $\lambda$, such that for all $\boldsymbol{\tau}_{h} \in X_{h}^{\Gamma}$ with $\mathbf{d i v}_{h} \boldsymbol{\tau}_{h}=\mathbf{0}$ it holds

$$
\left[\boldsymbol{\tau}_{h}, \boldsymbol{\tau}_{h}\right]_{X_{h}} \geq\left.\alpha\left|\| \boldsymbol{\tau}_{h}\right|\right|_{X_{h}} ^{2} .
$$

Proof. Let $\boldsymbol{\tau}_{h} \in X_{h}$. Given the tetrahedral mesh $\Omega_{h}^{\prime}$ introduced above, we define $\boldsymbol{\tau}_{h}^{\prime}$ in the space $V_{h}^{\Gamma}$ as follows. By definition of $\Omega_{h}^{\prime}$, each element $E \in \Omega_{h}$ is subdivided into tetrahedrons of $\Omega_{h}^{\prime}$; we call $V_{E}$ the restriction of $V_{h}^{\Gamma}$ to the local tetrahedral mesh in $E$. Furthermore, let $P_{E}$ indicate the space of piecewise constant vector fields on the same mesh with null integral on $E$. Given the space

$$
V_{E}^{N}=\left\{\boldsymbol{\delta}_{h}^{\prime} \in V_{E}:\left.\boldsymbol{\delta}_{h}^{\prime}\right|_{e} \cdot \mathbf{n}_{E}^{e}=\boldsymbol{\tau}_{E}^{e} \quad \forall e \in \partial E\right\},
$$

we solve the following local discrete problem on $E$

$$
\left\{\begin{array}{lr}
\text { Find } \boldsymbol{\tau}_{E}^{\prime} \in V_{E}^{N}, \mathbf{v}_{E}^{\prime} \in P_{E} \text { such that } & \\
\left(\operatorname{dev}\left(\boldsymbol{\tau}_{E}^{\prime}\right), \operatorname{dev}\left(\boldsymbol{\delta}_{E}^{\prime}\right)\right)_{L^{2}(E)}+\left(\mathbf{v}_{E}^{\prime}, \operatorname{div} \boldsymbol{\delta}_{E}^{\prime}\right)_{L^{2}(E)}=0 & \forall \boldsymbol{\delta}_{E}^{\prime} \in V_{E}^{0} \\
\left(\operatorname{div} \boldsymbol{\tau}_{E}^{\prime}, \mathbf{w}_{E}^{\prime}\right)_{L^{2}(E)}=0 & \forall \mathbf{w}_{E}^{\prime} \in P_{E},
\end{array}\right.
$$

where $V_{E}^{0}$ is the homogeneous version of $V_{E}^{N}$. The stability of the above problem follows from the same argument used for (3.3). Note that problem (3.12) is well posed since, due to the property $\boldsymbol{d i v}_{h} \boldsymbol{\tau}_{h}=\mathbf{0}$, the boundary data required in (3.11) is compatible with condition $(3.12)_{3}$.

The function $\left(\boldsymbol{\tau}_{E}^{\prime}-\frac{1}{3} \operatorname{tr}_{h}\left(\boldsymbol{\tau}_{h}\right) \mathbb{I}\right)$ is by definition in the space $V_{E}$ and clearly satisfies the discrete system (3.12), i.e.

$$
\begin{cases}\left(\operatorname{dev}\left(\boldsymbol{\tau}_{E}^{\prime}-\frac{1}{3} \operatorname{tr}_{h}\left(\boldsymbol{\tau}_{h}\right) \mathbb{I}\right), \operatorname{dev}\left(\boldsymbol{\delta}_{E}^{\prime}\right)\right)_{L^{2}(E)}+\left(\mathbf{v}_{E}^{\prime}, \operatorname{div} \boldsymbol{\delta}_{E}^{\prime}\right)_{L^{2}(E)}=0 & \forall \boldsymbol{\delta}_{E}^{\prime} \in V_{E}^{0} \\ \left(\operatorname{div}\left(\boldsymbol{\tau}_{E}^{\prime}-\frac{1}{3} \operatorname{tr}_{h}\left(\boldsymbol{\tau}_{h}\right) \mathbb{I}\right), \mathbf{w}_{E}^{\prime}\right)_{L^{2}(E)}=0 & \forall \mathbf{w}_{E}^{\prime} \in P_{E} .\end{cases}
$$

As a consequence, due to the stability of the problem, the function $\left(\boldsymbol{\tau}_{E}^{\prime}-\frac{1}{3} \operatorname{tr}_{h}\left(\boldsymbol{\tau}_{h}\right) \mathbb{I}\right)$ depends continuously on the boundary data. In particular, it can be proved that

$$
\left\|\boldsymbol{\tau}_{E}^{\prime}-\frac{1}{3} \operatorname{tr}_{h}\left(\boldsymbol{\tau}_{h}\right) \mathbb{I}\right\|_{L^{2}(E)}^{2} \lesssim \sum_{e \in \partial E} h_{e}\left\|\left(\boldsymbol{\tau}_{E}^{\prime}-\frac{1}{3} \operatorname{tr}_{h}\left(\boldsymbol{\tau}_{h}\right) \mathbb{I}\right) \cdot \mathbf{n}_{E}^{e}\right\|_{L^{2}(e)} .
$$


Let $\boldsymbol{\tau}_{h}^{\prime} \in V_{h}^{\Gamma}$ be the global tensor field in $H_{\text {div }}^{\Gamma}(\Omega)$ such that $\left.\boldsymbol{\tau}_{h}^{\prime}\right|_{E}=\boldsymbol{\tau}_{E}^{\prime}$ for all $E \in \Omega_{h}$. The boundary conditions and the continuity of the normal component across the faces of the triangulation are guaranteed by condition (3.11). Moreover, again due to (3.11), it holds that $\left(\boldsymbol{\tau}_{h}^{\prime}\right)_{I}=\boldsymbol{\tau}_{h}$ and $\left(\left(\boldsymbol{\tau}_{h}^{\prime}\right)_{I}\right)_{E}^{e}=\left.\boldsymbol{\tau}_{h}^{\prime}\right|_{e} \cdot \mathbf{n}_{E}^{e}$ for all $E \in \Omega_{h}, e \in \partial E$. Therefore, using (S1) we obtain

$$
\begin{aligned}
{\left[\boldsymbol{\tau}_{h}, \boldsymbol{\tau}_{h}\right]_{X_{h}} \gtrsim \sum_{E \in \Omega_{h}}\left\|\left|\boldsymbol{\tau}_{h}-\frac{1}{3} \operatorname{tr}_{h}\left(\boldsymbol{\tau}_{h}\right) \mathbb{I}_{I}\right|\right\|_{E}^{2} } & =\sum_{E \in \Omega_{h}}\|\|\left(\boldsymbol{\tau}_{h}^{\prime}-\frac{1}{3} \operatorname{tr}_{h}\left(\boldsymbol{\tau}_{h}\right) \mathbb{I}\right)_{I} \|_{E}^{2} \\
& =\sum_{E \in \Omega_{h}} \sum_{e \in \partial E} h_{e}\left\|\left(\boldsymbol{\tau}_{h}^{\prime}-\frac{1}{3} \operatorname{tr}_{h}\left(\boldsymbol{\tau}_{h}\right) \mathbb{I}\right) \cdot \mathbf{n}_{E}^{e}\right\|_{L^{2}(e)}^{2},
\end{aligned}
$$

which, using (3.14), gives

$$
\left[\boldsymbol{\tau}_{h}, \boldsymbol{\tau}_{h}\right]_{X_{h}} \gtrsim\left\|\boldsymbol{\tau}_{h}^{\prime}-\frac{1}{3} \operatorname{tr}_{h}\left(\boldsymbol{\tau}_{h}\right) \mathbb{I}\right\|_{L^{2}(\Omega)}^{2}
$$

From the $L^{2}$ orthogonality between deviatoric tensors and the identity $\mathbb{I}$, using (3.2) and (3.16), we have that

$$
\left[\boldsymbol{\tau}_{h}, \boldsymbol{\tau}_{h}\right]_{X_{h}} \gtrsim\left\|\operatorname{dev}\left(\boldsymbol{\tau}_{h}^{\prime}\right)\right\|_{L^{2}(\Omega)}^{2} \gtrsim\left\|\boldsymbol{\tau}_{h}^{\prime}\right\|_{L^{2}(\Omega)}^{2} .
$$

The final result follows from (3.17) through a scaling argument and recalling that $\boldsymbol{\tau}_{h}^{\prime} \cdot \mathbf{n}_{E}^{e}=\boldsymbol{\tau}_{E}^{e}$ for all $E \in \Omega_{h}$, $e \in \partial E$

$$
\left[\boldsymbol{\tau}_{h}, \boldsymbol{\tau}_{h}\right]_{X_{h}} \gtrsim \sum_{E \in \Omega_{h}} \sum_{e \in \partial E} h_{e}\left\|\boldsymbol{\tau}_{h}^{\prime} \cdot \mathbf{n}_{E}^{e}\right\|_{L^{2}(e)}=\|\| \boldsymbol{\tau}_{h} \mid \|_{X_{h}}^{2} .
$$

Combining the results of this section and the classical theory of [13] the following uniform stability lemma follows immediately.

Lemma 3.3. It exists one and only one solution to problem (2.27). Moreover it holds

$$
\left\|\left|\boldsymbol{\sigma}_{h}\right|\right\|_{X_{h}}+\left\|\mathbf{u}_{h}\right\|_{Q_{h}}+\left\|\mathbf{s}_{h}\right\|_{Q_{h}} \lesssim \sup _{\mathbf{v}_{h} \in Q_{h}} \frac{\left[\mathbf{f}_{I}, \mathbf{v}_{h}\right]_{Q_{h}}}{\left\|\mathbf{v}_{h}\right\|_{Q_{h}}} \leq\|\mathbf{f}\|_{L^{2}(\Omega)}
$$

where the constant $C$ is independent of $h, \lambda$.

\subsection{Convergence of the method}

In this section we show the convergence properties of the method. Due to the particular structure of the MFD method, the convergence does not follow from a straightforward application of the stability results of the previous section and some interpolation property. Before stating our result, we prove two preliminary lemmas.

Lemma 3.4. Let $\boldsymbol{\sigma}$ be the solution of (2.2). Then it holds

$$
\left\|\operatorname{as}_{h}\left(\boldsymbol{\sigma}_{I}\right)\right\|_{E} \lesssim h_{E}^{2}|\mathbf{f}|_{H^{1}(E)} \quad \forall E \in \Omega_{h} .
$$

Proof. Let $E \in \Omega_{h}$. Using an integration by parts and (2.1) 2 we get, for $i=1,2,3$,

$$
0=\int_{E}[\mathbf{a s}(\boldsymbol{\sigma})]_{i} \mathrm{~d} \mathbf{x}=\int_{E} \boldsymbol{\nabla} \psi_{E}^{i}: \boldsymbol{\sigma} \mathrm{d} \mathbf{x}=-\int_{E} \boldsymbol{\psi}_{E}^{i} \cdot \mathbf{f} \mathrm{d} \mathbf{x}+\sum_{e \in \partial E} \int_{e}\left(\boldsymbol{\sigma} \mathbf{n}_{E}^{e}\right) \cdot \boldsymbol{\psi}_{E}^{i} \mathrm{~d} \boldsymbol{\xi}
$$

where the $\boldsymbol{\psi}_{E}^{i}$ are defined in (2.14). From the definition of the operators (2.19) and (2.11) it follows

$$
\left[\mathbf{a s}_{h}\left(\boldsymbol{\sigma}_{I}\right)\right]_{i}=\frac{1}{|E|} \sum_{e \in \partial E} \int_{e}\left(\boldsymbol{\sigma}_{I}\right)_{E}^{e} \cdot \boldsymbol{\psi}_{E}^{i} \mathrm{~d} \boldsymbol{\xi}=\frac{1}{|E|} \sum_{e \in \partial E} \int_{e}\left(\boldsymbol{\sigma} \mathbf{n}_{E}^{e}\right) \cdot \boldsymbol{\psi}_{E}^{i} \mathrm{~d} \boldsymbol{\xi}
$$


Using (3.21) and recalling that $\int_{E} \psi_{E}^{i} \mathrm{~d} \mathbf{x}=0$, applying the Holder inequality and relation (3.22) give

$$
\left[\operatorname{as}_{h}\left(\boldsymbol{\sigma}_{I}\right)\right]_{i}=\frac{1}{|E|} \int_{E} \mathbf{f} \cdot \boldsymbol{\psi}_{E}^{i} \mathrm{~d} \mathbf{x}=\frac{1}{|E|} \int_{E}(\mathbf{f}-\overline{\mathbf{f}}) \cdot \boldsymbol{\psi}_{E}^{i} \mathrm{~d} \mathbf{x} \leq|E|^{-1 / 2}\|(\mathbf{f}-\overline{\mathbf{f}})\|_{L^{2}(E)}\left\|\boldsymbol{\psi}_{E}^{i}\right\|_{L^{\infty}(E)}
$$

where $\overline{\mathbf{f}}=\overline{\mathbf{f}}(E)$ denotes the average of $\mathbf{f}$ over the element $E$. Applying a standard interpolation estimate and noting that $\left\|\boldsymbol{\psi}_{E}^{i}\right\|_{L^{\infty}(E)} \lesssim h_{E}$ yields

$$
\left[\operatorname{as}_{h}\left(\boldsymbol{\sigma}_{I}\right)\right]_{i} \leq|E|^{-1 / 2} h_{E}^{2}|\mathbf{f}|_{H^{1}(E)} .
$$

The result follows from (3.24) and the definition of the local $Q_{h}$ norm $\|\cdot\|_{E}$ induced by (2.21).

Lemma 3.5. For all $\boldsymbol{\tau}_{h} \in X_{h}$ it holds that

$$
\left[\left(\mathbb{C} \varepsilon\left(\mathbf{p}^{1}\right)-\mathbb{C} \boldsymbol{\nabla} \mathbf{p}^{1}\right)_{I}, \boldsymbol{\tau}_{h}\right]_{E} \lesssim\left\|\boldsymbol{\nabla} \mathbf{p}^{1}\right\|_{L^{2}(E)}\left\|\operatorname{as}_{h}\left(\boldsymbol{\tau}_{h}\right)\right\|_{E},
$$

for all elements $E \in \Omega_{h}$ and all linear vector fields $\mathbf{p}^{1}$ in $E$.

Proof. A direct calculation shows that

$$
\boldsymbol{\varepsilon}\left(\mathbf{p}^{1}\right)-\boldsymbol{\nabla} \mathbf{p}^{1}=\frac{1}{2} \sum_{i=1}^{3}\left[\mathbf{a s}\left(\boldsymbol{\nabla} \mathbf{p}^{1}\right)\right]_{i} \boldsymbol{\nabla} \psi_{E}^{i}
$$

where we remind that the vector field $\mathbf{a s}\left(\boldsymbol{\nabla} \mathbf{p}^{1}\right)$ is constant over the element $E$. Therefore, by using the linearity of the scalar product, of the operator $\mathbb{C}$ and of the interpolant operator, we obtain that

$$
\left[\left(\mathbb{C} \varepsilon\left(\mathbf{p}^{1}\right)-\mathbb{C} \boldsymbol{\nabla} \mathbf{p}^{1}\right)_{I}, \boldsymbol{\tau}_{h}\right]_{E}=\frac{1}{2} \sum_{i=1}^{3}\left[\mathbf{a s}\left(\boldsymbol{\nabla} \mathbf{p}^{1}\right)\right]_{i}\left[\left(\mathbb{C} \boldsymbol{\nabla} \psi_{E}^{i}\right)_{I}, \boldsymbol{\tau}_{h}\right]_{E}
$$

Since the functions $\psi_{E}^{i}$ are linear, we can apply property (S2) to obtain

$$
\left[\left(\mathbb{C} \nabla \psi_{E}^{i}\right)_{I}, \boldsymbol{\tau}_{h}\right]_{E}=-\int_{E} \boldsymbol{\psi}_{E}^{i} \cdot \boldsymbol{d i v}_{h} \boldsymbol{\tau}_{h} \mathrm{~d} \mathbf{x}+\sum_{e \in \partial E} \int_{e} \boldsymbol{\tau}_{E}^{e} \cdot \boldsymbol{\psi}_{E}^{i} \mathrm{~d} \boldsymbol{\xi}=\sum_{e \in \partial E} \int_{e} \boldsymbol{\tau}_{E}^{e} \cdot \boldsymbol{\psi}_{E}^{i} \mathrm{~d} \boldsymbol{\xi}
$$

for $i=1,2,3$, where the last step follows because the integral of the $\boldsymbol{\psi}_{E}^{i}$ is null over the element $E$. Using (3.27), (3.28) and definition (2.19) gives

$$
\left[\left(\mathbb{C} \varepsilon\left(\mathbf{p}^{1}\right)-\mathbb{C} \boldsymbol{\nabla} \mathbf{p}^{1}\right)_{I}, \boldsymbol{\tau}_{h}\right]_{E}=\frac{|E|}{2} \operatorname{as}\left(\nabla \mathbf{p}^{1}\right) \cdot \operatorname{as}_{h}\left(\boldsymbol{\tau}_{h}\right)=\frac{1}{2} \int_{E} \operatorname{as}\left(\nabla \mathbf{p}^{1}\right) \cdot \operatorname{as}_{h}\left(\boldsymbol{\tau}_{h}\right) .
$$

The result follows from the Cauchy-Schwartz inequality and the definition of the operator as $(\cdot)$.

Proposition 3.2. It holds that

$$
\begin{aligned}
\|\left|\boldsymbol{\sigma}_{h}-\boldsymbol{\sigma}_{I}\right|||_{X_{h}} & \leq C h\left(\left\|\left.\mathbf{u}\right|_{H^{2}(\Omega)}+\right\| \boldsymbol{\sigma} \|_{H^{1}(\Omega)}+|\mathbf{f}|_{H^{1, h}(\Omega)}\right) \\
\operatorname{div}_{h} \boldsymbol{\sigma}_{h} & =(\operatorname{div} \boldsymbol{\sigma})_{I},
\end{aligned}
$$

where the constant $C$ is independent of $h, \lambda$ and $|\mathbf{f}|_{H^{1, h}(\Omega)}^{2}=\sum_{E \in \Omega_{h}}|\mathbf{f}|_{H^{1}(E)}^{2}$. 
Proof. From (2.27) 2 and (2.13), (2.2) 2 it follows that

$$
\operatorname{div}_{h} \sigma_{h}=\mathbf{f}_{I}, \quad \operatorname{div}_{h} \sigma_{I}=(\operatorname{div} \boldsymbol{\sigma})_{I}=\mathbf{f}_{I},
$$

which in particular proves (3.30) ${ }_{2}$. Due to (3.31) we also have that $\boldsymbol{d i v}_{h}\left(\boldsymbol{\sigma}_{h}-\boldsymbol{\sigma}_{I}\right)=\mathbf{0}$ and therefore we can apply Lemma 3.2 and obtain

$$
\left\|\left|\sigma_{h}-\sigma_{I}\right|\right\|_{X_{h}}^{2} \lesssim\left[\sigma_{h}-\sigma_{I}, \sigma_{h}-\sigma_{I}\right]_{X_{h}} .
$$

From the above bound, using $(2.27)_{1},(2.1)$ and again that $\boldsymbol{d i v}_{h}\left(\boldsymbol{\sigma}_{h}-\boldsymbol{\sigma}_{I}\right)=\mathbf{0}$ gives

$$
\begin{aligned}
\left\|\boldsymbol{\sigma}_{h}-\boldsymbol{\sigma}_{I} \mid\right\|_{X_{h}}^{2} & \lesssim\left[\boldsymbol{\sigma}_{I}-\boldsymbol{\sigma}_{h}, \boldsymbol{\sigma}_{I}\right]_{X_{h}}+\left[\mathbf{s}_{h}, \operatorname{as}_{h}\left(\boldsymbol{\sigma}_{I}-\boldsymbol{\sigma}_{h}\right)\right]_{Q_{h}} \\
& =\left[\boldsymbol{\sigma}_{I}-\boldsymbol{\sigma}_{h},(\mathbb{C} \varepsilon(\mathbf{u}))_{I}\right]_{X_{h}}+\left[\mathbf{s}_{h}, \operatorname{as}_{h}\left(\boldsymbol{\sigma}_{I}-\boldsymbol{\sigma}_{h}\right)\right]_{Q_{h}} .
\end{aligned}
$$

Now, let $\mathbf{u}^{1}$ be the unique piecewise linear vector field on $\Omega_{h}$ that satisfies

$$
\int_{E} \mathbf{u}^{1} \mathrm{~d} \mathbf{x}=\int_{E} \mathbf{u} \mathrm{d} \mathbf{x}, \quad \int_{E} \boldsymbol{\nabla} \mathbf{u}^{1} \mathrm{~d} \mathbf{x}=\int_{E} \boldsymbol{\nabla} \mathbf{u} \mathrm{d} \mathbf{x}
$$

for all $E \in \Omega_{h}$. Classical bounds and interpolation estimates give

$$
\begin{aligned}
\left\|\boldsymbol{\nabla} \mathbf{u}^{1}\right\|_{L^{2}(E)} & \lesssim\|\nabla \mathbf{\nabla u}\|_{L^{2}(E)}, \\
h_{E}^{-1}\left\|\mathbf{u}-\mathbf{u}^{1}\right\|_{L^{2}(E)}+\left\|\boldsymbol{\nabla u}-\boldsymbol{\nabla} \mathbf{u}^{1}\right\|_{L^{2}(E)} & \lesssim h_{E}\|\mathbf{u}\|_{H^{2}(E)} .
\end{aligned}
$$

Noting that $\mathbf{a s}_{h} \boldsymbol{\sigma}_{h}$ is zero due to $(2.27)_{3}$ and adding and subtracting terms, we get

$$
\begin{aligned}
\left\|\left|\boldsymbol{\sigma}_{h}-\boldsymbol{\sigma}_{I}\right|\right\|_{X_{h}}^{2} \lesssim\left[\boldsymbol{\sigma}_{I}-\boldsymbol{\sigma}_{h},(\mathbb{C} \varepsilon(\mathbf{u})-\right. & \left.\left.\mathbb{C} \varepsilon\left(\mathbf{u}^{1}\right)\right)_{I}\right]_{X_{h}}+\left[\boldsymbol{\sigma}_{I}-\boldsymbol{\sigma}_{h},\left(\mathbb{C} \varepsilon\left(\mathbf{u}^{1}\right)-\mathbb{C} \boldsymbol{\nabla} \mathbf{u}^{1}\right)_{I}\right]_{X_{h}} \\
& +\left[\boldsymbol{\sigma}_{I}-\boldsymbol{\sigma}_{h},\left(\mathbb{C} \nabla \mathbf{u}^{1}\right)_{I}\right]_{X_{h}}+\left[\mathbf{s}_{h}, \mathbf{a s}_{h}\left(\boldsymbol{\sigma}_{I}\right)\right]_{Q_{h}}=T_{1}+T_{2}+T_{3}+T_{4} .
\end{aligned}
$$

We now bound the four terms $T_{1}, \ldots, T_{4}$ introduced above. From (3.33) it follows that

$$
\int_{E} \mathbb{C} \varepsilon\left(\mathbf{u}^{1}\right) \mathrm{d} \mathbf{x}=\int_{E} \mathbb{C} \varepsilon(\mathbf{u}) \mathrm{d} \mathbf{x} .
$$

Thus, since due to (2.2) it holds $\boldsymbol{\sigma}=\mathbb{C} \varepsilon(\mathbf{u})$, we can write

$$
\mathbb{C} \varepsilon\left(\mathbf{u}^{1}\right)=\overline{\boldsymbol{\sigma}}
$$

where $\bar{\sigma}$ is a piecewise constant tensor field given by the componentwise average of $\boldsymbol{\sigma}$. Combining the above argument and assumption (S1) yields

$$
T_{1} \leq||\left|\boldsymbol{\sigma}_{h}-\boldsymbol{\sigma}_{I}\right|||_{X_{h}}||\left|(\boldsymbol{\sigma}-\overline{\boldsymbol{\sigma}})_{I}\right| \|_{X_{h}} .
$$

The interpolation operator (2.11) restricted to faces is an $L^{2}$ projection of the normal component. As a consequence, it holds

$$
\left\|\left|(\boldsymbol{\sigma}-\overline{\boldsymbol{\sigma}})_{I}\right|\right\|_{E}^{2}=\sum_{e \in \partial E} h_{e}\left\|(\boldsymbol{\sigma}-\overline{\boldsymbol{\sigma}})_{I}\right\|_{L^{2}(e)}^{2} \leq \sum_{e \in \partial E} h_{e}\|\boldsymbol{\sigma}-\overline{\boldsymbol{\sigma}}\|_{L^{2}(e)}^{2} .
$$

Now, using an Agmon inequality [1], a standard interpolation estimate, and noting that $h_{e} \leq h_{E} \forall e \in \partial E$, from bound (3.39) it follows that

$$
\left.||\left|(\boldsymbol{\sigma}-\overline{\boldsymbol{\sigma}})_{I}\right|\right|_{E} ^{2} \lesssim \| \boldsymbol{\sigma}-\left.\overline{\boldsymbol{\sigma}}\right|_{L^{2}(E)} ^{2}+h_{E}^{2}|\boldsymbol{\sigma}-\overline{\boldsymbol{\sigma}}|_{H^{1}(E)}^{2} \lesssim h_{E}^{2}|\boldsymbol{\sigma}|_{H^{1}(E)}^{2} .
$$


Joining the bounds (3.38) and (3.40) gives

$$
\left.T_{1} \lesssim h||\left|\boldsymbol{\sigma}_{h}-\boldsymbol{\sigma}_{I}\right|\right|_{X_{h}}|\boldsymbol{\sigma}|_{H^{1}(\Omega)}
$$

For the second term in (3.35), first using Lemma 3.5 and $(2.27)_{3}$, then due to Lemma 3.4 and bound $(3.34)_{2}$, it follows

$$
\begin{aligned}
T_{2} & \lesssim \sum_{E \in \Omega_{h}}\left\|\nabla \mathbf{u}^{1}\right\|_{L^{2}(E)}\left\|\operatorname{as}_{h}\left(\boldsymbol{\sigma}_{I}-\boldsymbol{\sigma}_{h}\right)\right\|_{E}=\sum_{E \in \Omega_{h}}\left\|\nabla \mathbf{u}^{1}\right\|_{L^{2}(E)}\left\|\operatorname{as}_{h}\left(\boldsymbol{\sigma}_{I}\right)\right\|_{E} \\
& \lesssim \sum_{E \in \Omega_{h}} h_{E}^{2}\|\boldsymbol{\nabla u}\|_{L^{2}(E)}|\mathbf{f}|_{H^{1}(E)} \lesssim h^{2}\|\boldsymbol{\nabla u}\|_{L^{2}(\Omega)}\|\mathbf{f}\|_{H^{1, h}(\Omega)}
\end{aligned}
$$

For the third term in (3.35), using assumption (S2) and recalling that $\operatorname{div}_{h}\left(\boldsymbol{\sigma}_{I}-\boldsymbol{\sigma}_{h}\right)=0$, yields

$$
T_{3}=\sum_{E \in \Omega_{h}}\left[\boldsymbol{\sigma}_{I}-\boldsymbol{\sigma}_{h},\left(\mathbb{C} \boldsymbol{\nabla} \mathbf{u}^{1}\right)_{I}\right]_{E}=\sum_{E \in \Omega_{h}} \sum_{e \in \partial E} \int_{e}\left(\boldsymbol{\sigma}_{I}-\boldsymbol{\sigma}_{h}\right)_{E}^{e} \cdot \mathbf{u}^{1} \mathrm{~d} \boldsymbol{\xi} .
$$

Let $E^{\prime}$ and $E^{\prime \prime}$ indicate the two elements that share any internal face $e$, where the normal $\mathbf{n}_{e}$ points from $E^{\prime}$ to $E^{\prime \prime}$. Then, for any sufficiently regular vector field $\mathbf{g}$ on $\Omega$, we define

$$
\llbracket \mathbf{g} \rrbracket_{e}=\mathbf{g}^{\prime}-\mathbf{g}^{\prime \prime}
$$

where $\mathbf{g}^{\prime}$ is the trace of $\left.\mathbf{g}\right|_{E^{\prime}}$ on $e$, and $\mathbf{g}^{\prime \prime}$ is the trace of $\left.\mathbf{g}\right|_{E^{\prime \prime}}$ on $e$. Due to (2.10) and (3.43), recalling that by definition $\left(\boldsymbol{\sigma}_{h}\right)_{e}=\left(\boldsymbol{\sigma}_{I}\right)_{e}=0$ on all faces $e \in \Gamma$, we can write

$$
T_{3}=\sum_{e \in \Sigma_{h}^{\prime}} \int_{e}\left(\boldsymbol{\sigma}_{I}-\boldsymbol{\sigma}_{h}\right)_{e} \cdot \llbracket \mathbf{u}^{1} \rrbracket_{e} \mathrm{~d} \boldsymbol{\xi}+\sum_{e \in \Gamma^{\prime}} \int_{e}\left(\boldsymbol{\sigma}_{I}-\boldsymbol{\sigma}_{h}\right)_{e} \cdot \mathbf{u}^{1} \mathrm{~d} \boldsymbol{\xi}
$$

where $\Sigma_{h}^{\prime} \subset \Sigma_{h}$ indicates the set of internal faces. Using that, by definition, $\llbracket \mathbf{u} \rrbracket_{e}=0 \forall e \in \Sigma_{h}^{\prime}$ and $\mathbf{u}=0$ on $\Gamma^{\prime}$, identity (3.44) becomes

$$
T_{3}=\sum_{e \in \Sigma_{h}^{\prime}} \int_{e}\left(\boldsymbol{\sigma}_{I}-\boldsymbol{\sigma}_{h}\right)_{e} \cdot \llbracket \mathbf{u}^{1}-\mathbf{u} \rrbracket_{e} \mathrm{~d} \boldsymbol{\xi}+\sum_{e \in \Gamma^{\prime}} \int_{e}\left(\boldsymbol{\sigma}_{I}-\boldsymbol{\sigma}_{h}\right)_{e} \cdot\left(\mathbf{u}^{1}-\mathbf{u}\right) \mathrm{d} \boldsymbol{\xi} .
$$

For all $e \in \Gamma^{\prime}$, first due to a Cauchy-Schwartz inequality, then using as above an Agmon inequality and standard interpolation estimates, it easily follows

$$
\begin{aligned}
\int_{e}\left(\boldsymbol{\sigma}_{I}-\boldsymbol{\sigma}_{h}\right)_{e} \cdot\left(\mathbf{u}^{1}-\mathbf{u}\right) \mathrm{d} \boldsymbol{\xi} & \leq\left\|\left(\boldsymbol{\sigma}_{I}-\boldsymbol{\sigma}_{h}\right)_{e}\right\|_{L^{2}(e)}\left\|\mathbf{u}^{1}-\mathbf{u}\right\|_{L^{2}(e)} \\
& \lesssim\left\|\left(\boldsymbol{\sigma}_{I}-\boldsymbol{\sigma}_{h}\right)_{e}\right\|_{L^{2}(e)} h_{E_{e}}^{3 / 2}|\mathbf{u}|_{H^{2}\left(E_{e}\right)}
\end{aligned}
$$

where $E_{e}$ is the element pertaining to the boundary face $e$. Applying bound (3.46) in the second term of (3.45) and using a Cauchy-Schwartz inequality gives

$$
\begin{aligned}
\sum_{e \in \Gamma^{\prime}} \int_{e}\left(\boldsymbol{\sigma}_{I}-\boldsymbol{\sigma}_{h}\right)_{e} \cdot\left(\mathbf{u}^{1}-\mathbf{u}\right) \mathrm{d} \boldsymbol{\xi} & \lesssim\left(\sum_{e \in \Gamma^{\prime}} h_{E_{e}}||\left(\boldsymbol{\sigma}_{I}-\boldsymbol{\sigma}_{h}\right)_{e} \|_{L^{2}(e)}^{2}\right)^{1 / 2}\left(\sum_{e \in \Gamma^{\prime}} h_{E_{e}}^{2}|\mathbf{u}|_{H^{2}\left(E_{e}\right)}^{2}\right)^{1 / 2} \\
& \lesssim h\left|\left\|\boldsymbol{\sigma}_{I}-\left.\boldsymbol{\sigma}_{h}\left|\|_{X_{h}}\right| \mathbf{u}\right|_{H^{2}(\Omega)},\right.\right.
\end{aligned}
$$


where we also used that $h_{e} \sim h_{E_{e}}$ due to (M3). The first term in (3.45) is bounded with an identical argument, simply splitting the two side contributions in the jumps. One finally obtains

$$
T_{3} \lesssim h||\left|\boldsymbol{\sigma}_{I}-\boldsymbol{\sigma}_{h}\right|||_{X_{h}}|\mathbf{u}|_{H^{2}(\Omega)} .
$$

Furthermore, combining a Cauchy-Schwartz estimate for the $Q_{h}$ scalar product with Lemma 3.4 and inequality (3.19) gives

$$
T_{4} \lesssim\left\|\mathbf{s}_{h}\right\|_{Q_{h}}\left\|\operatorname{as}_{h}\left(\boldsymbol{\sigma}_{I}\right)\right\|_{Q_{h}} \lesssim h_{E}^{2}|\mathbf{f}|_{H^{1, h}(\Omega)}^{2}
$$

Joining (3.35) with the bounds (3.41), (3.42), (3.48), (3.49), and applying some simple algebra immediately leads to the desired result.

Note that the second bound in Proposition 3.2 implies in particular

$$
\left\|\operatorname{div}_{h} \boldsymbol{\sigma}_{h}-\operatorname{div} \boldsymbol{\sigma}\right\|_{Q_{h}} \lesssim h|\mathbf{f}|_{H^{1, h}(\Omega)} .
$$

The regularity required for the solution in Proposition 3.2 is the standard one for finite element methods converging with linear order. Such regularity holds on all convex domains whenever $\mathbf{f} \in L^{2}(\Omega)$, see for instance Theorem 2.1 in [3]. In addition, we are assuming $\mathbf{f} \in H^{1}(E)$ for all $E \in \Omega$. Nevertheless, this condition is expected to be satisfied for most problems, since it admits jumps of the load across the mesh faces and one can adapt the mesh to the load.

Once the previous results of this section are established, the convergence properties for the displacements follow easily by combining the steps of [16], Theorems 5.5 and 5.6, and the techniques developed above for the stress variable. Therefore we do not show the proof of the following propositions.

Proposition 3.3. Let $\Omega$ be convex. It holds

$$
\left\|\mathbf{u}_{h}-\mathbf{u}_{I}\right\|_{Q_{h}} \leq C h\left(|| \mathbf{u} \|_{H^{2}(\Omega)}+|\mathbf{f}|_{H^{1, h}(\Omega)}\right)
$$

where the constant $C$ is independent of $h$ and $\lambda$.

Under the assumption of the existence of a local lifting operator $R_{E}$, as in Theorem 5.1 of [16], we also have a superconvergence property. Given $E \in \Omega_{h}$, let $R_{E}$ denote a lifting operator acting on $\left.X_{h}\right|_{E}$ with values in $L^{2}(E)$ that satisfies

$$
\begin{aligned}
R_{E}\left(\left.\boldsymbol{\tau}_{h}\right|_{E}\right) \cdot \mathbf{n}_{e} & =\boldsymbol{\tau}_{e} & & \text { on } \partial E \\
\operatorname{div} R_{E}\left(\left.\boldsymbol{\tau}_{h}\right|_{E}\right) & =\left.\left(\operatorname{div}_{h} \boldsymbol{\tau}_{h}\right)\right|_{E} & & \text { in } E \\
{\left[\boldsymbol{\tau}_{h}, \boldsymbol{\delta}_{h}\right]_{E} } & =\int_{E} \mathbb{C}^{-1} R_{E} \boldsymbol{\tau}_{h}: R_{E} \boldsymbol{\delta}_{h} \mathrm{~d} \mathbf{x} & &
\end{aligned}
$$

for all $\boldsymbol{\tau}_{h}, \boldsymbol{\delta}_{h}$ in $X_{h}$. Moreover, for all constant tensor fields $\boldsymbol{\theta}$ on $E$, we have that $R_{E}\left(\boldsymbol{\theta}_{I}\right)=\boldsymbol{\theta}$.

Proposition 3.4. Let $R_{E}$ as above exist for all $E \in \Omega$. Then it holds

$$
\left\|\mathbf{u}_{h}-\mathbf{u}_{I}\right\|_{Q_{h}} \leq C h^{1+s}\left(\|\left.\mathbf{u}\right|_{H^{2}(\Omega)}+|\mathbf{f}|_{H^{1, h}(\Omega)}\right),
$$

where the constant $C$ is independent of $h, \lambda$ and $0 \leq s \leq 1$ depends on the domain $\Omega$ and diffusivity tensor field $\mathbb{C}$. In particular, $s=1$ if $\Omega$ is convex and $\mathbb{C}$ constant over the domain.

\section{Construction of a scalar product}

In this section we show that the scalar product (2.23), which satisfies properties (S1) and (S2), can be built by following the same algebraic construction developed for the diffusion problem in [17] and extended in [8] to the higher order case. We limit our presentation to the strictly necessary definitions, and refer to the above 
papers for a more detailed description of the procedure. The main results of this section are Lemma 4.2 and Proposition 4.1.

In order to construct the scalar product (2.23), it is sufficient to define the local forms $[\cdot, \cdot]_{E}, E \in \Omega_{h}$. Given any $\boldsymbol{\tau}_{h} \in X_{h}$, let

$$
\boldsymbol{\tau}_{E}=\left\{\boldsymbol{\tau}_{E}^{e}\right\}_{e \in \partial E}
$$

be the collection of the local face values, see Section 2.2.2. We also identify $\boldsymbol{\tau}_{E}$ with a vector in $\mathbb{R}^{9 n}$, where $n=n(E)$ is equal to the number of faces of $E$. Following (2.8), the components of the vector $\boldsymbol{\tau}_{E}$ are ordered in the following format

$$
\boldsymbol{\tau}_{E}=\left[\widehat{\boldsymbol{\tau}}_{e_{1}}, \widehat{\boldsymbol{\tau}}_{e_{2}}, \ldots, \widehat{\boldsymbol{\tau}}_{e_{n}}, \widetilde{\boldsymbol{\tau}}_{e_{1}}^{1}, \widetilde{\boldsymbol{\tau}}_{e_{2}}^{1}, \ldots, \widehat{\boldsymbol{\tau}}_{e_{n}}^{1}, \widetilde{\boldsymbol{\tau}}_{e_{1}}^{2}, \widetilde{\boldsymbol{\tau}}_{e_{2}}^{2}, \ldots, \widehat{\boldsymbol{\tau}}_{e_{n}}^{2}\right]^{T},
$$

where we numbered the faces $e_{1}, e_{2}, \ldots, e_{n}$ of $E$.

Then, we need to build matrices $\mathrm{M}_{E} \in \mathbb{R}^{9 n \times 9 n}$ such that the local scalar products

$$
\left[\boldsymbol{\tau}_{E}, \boldsymbol{\delta}_{E}\right]_{E}=\boldsymbol{\tau}_{E}^{T} \mathrm{M}_{E} \boldsymbol{\delta}_{E} \quad \forall \boldsymbol{\tau}_{E},\left.\boldsymbol{\delta}_{E} \in X_{h}\right|_{E}
$$

are definite positive and satisfy assumptions (S1) and (S2).

Let $\mathbf{p}_{j}^{1}, j=1,2, \ldots, 9$, be a basis for the space of functions in $\left[\mathcal{P}_{1}(E)\right]^{3}$ with zero average on $E$. We define $\mathbf{N}$ as the matrix in $\mathbb{R}^{9 n \times 9}$ whose $j$-th column is given by the degrees-of-freedom of $\left(\mathbb{C} \nabla \mathbf{p}_{j}^{1}\right)_{I}$ on the faces of $E$

$$
\left.\mathrm{N}\right|_{j}=\left(\left(\mathbb{C} \nabla \mathbf{p}_{j}^{1}\right)_{I}\right)_{E}, \quad j=1,2, \ldots, 9,
$$

ordered as in (4.2). Note that, since the $\left(\mathbb{C} \boldsymbol{\nabla} \mathbf{p}_{j}^{1}\right)$ are constant over the element, the last $6 n$ components of the vectors $\left.\mathbf{N}\right|_{j}, j=1,2, \ldots, 9$, vanish. Therefore only the first $3 n$ rows of the matrix $\mathbf{N}$ are non zero and must be effectively computed. Let $\mathrm{R}$ be the matrix in $\mathbb{R}^{9 n \times 9}$ defined by

$$
\left.\mathrm{R}\right|_{j}=\left(\begin{array}{l}
\left.\mathrm{R}_{0}\right|_{j} \\
\left.\mathrm{R}_{1}^{1}\right|_{j} \\
\left.\mathrm{R}_{1}^{2}\right|_{j}
\end{array}\right), \quad j=1,2, \ldots, 9
$$

where

$$
\left.\mathrm{R}_{0}\right|_{j}=\left(\begin{array}{c}
\int_{e_{1}} \mathbf{p}_{j}^{1} \mathrm{~d} \boldsymbol{\xi} \\
\vdots \\
\int_{e_{n}} \mathbf{p}_{j}^{1} \mathrm{~d} \boldsymbol{\xi}
\end{array}\right),\left.\quad \mathbf{R}_{1}^{i}\right|_{j}=\left(\begin{array}{c}
\int_{e_{1}} \frac{\xi_{i}}{h_{e_{1}}} \mathbf{p}_{j}^{1} \mathrm{~d} \boldsymbol{\xi} \\
\vdots \\
\int_{e_{n}} \frac{\xi_{i}}{h_{e_{n}}} \mathbf{p}_{j}^{1} \mathrm{~d} \boldsymbol{\xi}
\end{array}\right), \quad i=1,2
$$

Following the same proof as in $[8,17]$, we have

$$
\mathrm{N}^{T} \mathrm{R}=|E| \overline{\mathrm{K}}
$$

where the symmetric and definite positive matrix $\overline{\mathrm{K}} \in \mathbb{R}^{9 \times 9}$ is defined by

$$
\overline{\mathrm{K}}_{i j}=\frac{1}{|E|} \int_{E} \mathbb{C} \boldsymbol{\nabla} \mathbf{p}_{i}^{1}: \nabla \mathbf{p}_{j}^{1} \mathrm{~d} \mathbf{x}, \quad i, j=1,2, \ldots, 9 .
$$

Given the orthogonal decomposition

$$
\mathbb{R}^{9 n}=\operatorname{img}(\mathrm{N}) \oplus \operatorname{img}(\mathrm{N})^{\perp}=\operatorname{img}(\mathrm{N}) \oplus \operatorname{ker}(\mathrm{N}),
$$

we define $\mathrm{C} \in \mathbb{R}^{9 n \times(9 n-9)}$ as the matrix with columns given by a orthonormal basis for $\operatorname{ker}(\mathrm{N})$. Then, we define our symmetric local matrices $\mathrm{M}_{E}$ by

$$
\mathrm{M}_{E}=\mathrm{M}_{1}+\mathrm{M}_{2}, \quad \mathrm{M}_{1}=\mathrm{R} \frac{\overline{\mathrm{K}}^{-1}}{|E|} \mathrm{R}^{T}, \mathrm{M}_{2}=\mathrm{CUC}^{T},
$$


where $U \in \mathbb{R}^{(9 n-9) \times(9 n-9)}$ is any positive definite matrix with correct scaling properties with respect to the element size and material parameter $\mu$. A good choice is given by

$$
\mathrm{U}=\frac{|E|}{\mu_{E}} \mathrm{I}_{\mathrm{d}}
$$

where $\mu_{E}$ is the value of $\mu$ on $E$, and $\mathrm{I}_{\mathbf{d}} \in \mathbb{R}^{(9 n-9) \times(9 n-9)}$ the identity matrix.

As already mentioned, the algebraic construction given above is a multiple component version of the high order one developed for the diffusion problem in [8]. Furthermore, the present procedure is simpler in the sense that we need to satisfy the consistency assumption (S2) only for linear polynomials instead of second order ones, see also Remark 4.1. As a consequence, we automatically inherit a part of the results already developed in [8] for the diffusion problem.

More precisely, the consistency property (S2), the boundedness (2.24) in (S1) and the (non uniform in $\lambda$ ) positivity of the scalar product follow from the same arguments of [8]. Moreover, the following lemma is directly inherited from the results of the paper mentioned above.

Lemma 4.1. It holds

$$
\mathbf{w}^{T} \mathrm{M}_{1} \mathbf{w} \leq C_{1} \frac{|E|}{\mu_{E}}\|\mathbf{w}\|^{2} \quad \forall \mathbf{w} \in \mathbb{R}^{9 n},
$$

with $C_{1}$ depending only on the constants in (M1)-(M5).

Therefore, we are left to prove property (2.25), which stems from the non uniform coercivity of the elasticity tensor $\mathbb{C}^{-1}$ with respect to $\lambda$, see (2.5). Due to this lack of uniform coercivity on the whole space, this condition is more involved than its counterpart in [8].

We start noting that, due to definition (2.16), the value of the trace operator $\operatorname{tr}_{h}$ in $E$ only depends on the local quantity $\boldsymbol{\tau}_{E}$ defined in (4.1). In other words, we have that $\operatorname{tr}_{h}\left(\boldsymbol{\tau}_{h}\right)_{E}=\operatorname{tr}_{E}\left(\boldsymbol{\tau}_{E}\right)$ for all $\boldsymbol{\tau}_{h} \in X_{h}$, where the local trace operator is given by

$$
\operatorname{tr}_{E}\left(\boldsymbol{\delta}_{E}\right)=\left.\frac{1}{|E|} \sum_{e \in \partial E} \int_{e} \boldsymbol{\delta}_{E}^{e} \cdot \boldsymbol{\varphi}_{E} \mathrm{~d} \boldsymbol{\xi} \quad \forall \boldsymbol{\delta}_{E} \in X_{h}\right|_{E},
$$

and $\boldsymbol{\varphi}_{E}$ is defined in (2.14). Analogously, with a little abuse of notation we can write $\left\|\left|\boldsymbol{\tau}_{h}\right|\right\|\left\|_{E}=\right\|\left|\boldsymbol{\tau}_{E}\right| \|\left.\right|_{E}$ for all $\tau_{h} \in X_{h}$, where

$$
\left.\left\|\boldsymbol{\delta}_{E}\right\|\right|_{E} ^{2}=\left.\sum_{e \in \partial E} h_{e}\left\|\boldsymbol{\delta}_{E}^{e}\right\|_{L^{2}(e)}^{2} \quad \forall \boldsymbol{\delta}_{E} \in X_{h}\right|_{E}
$$

We can now state the following lemma.

Lemma 4.2. It exists a positive constant $C_{+}$, independent of $h, \lambda, \mu$, such that

$$
\boldsymbol{\delta}_{E}^{T} \mathrm{M}_{1} \boldsymbol{\delta}_{E}=\left[\boldsymbol{\delta}_{E}, \boldsymbol{\delta}_{E}\right]_{E} \geq \frac{C_{+}}{\mu_{E}}\|\| \boldsymbol{\delta}_{E}-\frac{1}{3} \operatorname{tr}_{E}\left(\boldsymbol{\delta}_{E}\right) \mathbb{I}_{E}\|\|_{E}^{2}
$$

for all $\boldsymbol{\delta}_{E} \in \operatorname{img}(\mathrm{N})$, where $\mathbb{I}_{E}=\left(\mathbb{I}_{I}\right)_{E}$.

Proof. The first identity in (4.14) follows immediately from (4.3), (4.10) and the definition of C. Moreover, since $\boldsymbol{\delta}_{E} \in \operatorname{img}(\mathbf{N})$, it exists $\mathbf{w} \in \mathbb{R}^{9}$ such that

$$
\boldsymbol{\delta}_{E}=\mathrm{Nw}=\left.\sum_{j=1}^{9} w_{j} \mathrm{~N}\right|_{j}=\sum_{j=1}^{9} w_{j}\left(\left(\mathbb{C} \boldsymbol{\nabla} \mathbf{p}_{j}^{1}\right)_{I}\right)_{E}=\left(\left(\mathbb{C} \boldsymbol{\nabla} \mathbf{w}^{1}\right)_{I}\right)_{E}
$$


where the linear function

$$
\mathbf{w}^{1}=\sum_{j=1}^{9} w_{j} \mathbf{p}_{j}^{1}
$$

Using (4.10) and (4.7) from (4.15) we get

$$
\begin{aligned}
\boldsymbol{\delta}_{E}^{T} \mathrm{M}_{1} \boldsymbol{\delta}_{E} & =\mathbf{w}^{T} \mathrm{~N}^{T} \mathrm{R} \frac{\overline{\mathrm{K}}^{-1}}{|E|} \mathrm{R}^{T} \mathrm{~N} \mathbf{w}=|E| \mathbf{w}^{T} \overline{\mathrm{K}} \mathbf{w}=\sum_{i=1}^{9} \sum_{j=1}^{9} w_{i} w_{j} \int_{E} \mathbb{C} \boldsymbol{\nabla} \mathbf{p}_{i}^{1}: \nabla \mathbf{p}_{j}^{1} \mathrm{~d} \mathbf{x} \\
& =\int_{E} \mathbb{C} \boldsymbol{\nabla} \mathbf{w}^{1}: \boldsymbol{\nabla} \mathbf{w}^{1} \mathrm{~d} \mathbf{x} .
\end{aligned}
$$

Combining bound (4.17) with the definition of the elastic tensor $\mathbb{C}$ on $E$

$$
\mathbb{C} \boldsymbol{\theta}=2 \mu_{E} \boldsymbol{\theta}+\lambda_{E} \operatorname{tr}(\boldsymbol{\theta}) \mathbb{I} \quad \forall \boldsymbol{\theta} \in \mathbb{R}^{3 \times 3}
$$

we get

$$
\boldsymbol{\delta}_{E}^{T} \mathrm{M}_{1} \boldsymbol{\delta}_{E}=2 \mu_{E}\left\|\nabla \mathbf{w}^{1}\right\|_{L^{2}(E)}^{2}+\lambda_{E}\left\|\operatorname{div} \mathbf{w}^{1}\right\|_{L^{2}(E)}^{2} \geq 2 \mu_{E}\left\|\nabla \mathbf{w}^{1}\right\|_{L^{2}(E)}^{2},
$$

where $\mu_{E}, \lambda_{E}$ are the values of $\mu, \lambda$ on the element $E$.

Due to (4.13) and (4.15), the definition of interpolant (2.11) and an integration by parts give

$$
\begin{aligned}
\operatorname{tr}_{E}\left(\boldsymbol{\delta}_{E}\right) & =\frac{1}{|E|} \sum_{e \in \partial E} \int_{e}\left(\left(\mathbb{C} \boldsymbol{\nabla} \mathbf{w}^{1}\right)_{I}\right)_{E}^{e} \cdot \boldsymbol{\varphi}_{E} \mathrm{~d} \boldsymbol{\xi}=\frac{1}{|E|} \sum_{e \in \partial E} \int_{e}\left(\mathbb{C} \boldsymbol{\nabla} \mathbf{w}^{1}\right) \mathbf{n}_{E}^{e} \cdot \boldsymbol{\varphi}_{E} \mathrm{~d} \boldsymbol{\xi} \\
& =\frac{1}{|E|} \int_{E}\left(\mathbb{C} \boldsymbol{\nabla} \mathbf{w}^{1}\right): \boldsymbol{\nabla} \boldsymbol{\varphi}_{E} \mathrm{~d} \mathbf{x}=\frac{1}{|E|} \int_{E} \operatorname{tr}\left(\mathbb{C} \boldsymbol{\nabla} \mathbf{w}^{1}\right) \mathrm{d} \mathbf{x} \\
& =\operatorname{tr}\left(\mathbb{C} \boldsymbol{\nabla} \mathbf{w}^{1}\right)=\left(2 \mu_{E}+3 \lambda_{E}\right) \operatorname{div} \mathbf{w}^{1}
\end{aligned}
$$

where we used that $\operatorname{div}\left(\mathbb{C} \nabla \mathbf{w}^{1}\right)=0$ since $\mathbf{w}^{1}$ is linear.

Using (4.15) and (4.19) we now have

$$
\begin{aligned}
\|\| \boldsymbol{\delta}_{E}-\frac{1}{3} \operatorname{tr}_{E}\left(\boldsymbol{\delta}_{E}\right) \mathbb{I}_{E} \mid \|_{E}^{2} & =\left\|\left(\left(\mathbb{C} \nabla \mathbf{w}^{1}-\frac{1}{3}\left(2 \mu_{E}+3 \lambda_{E}\right) \operatorname{div} \mathbf{w}^{1} \mathbb{I}\right)_{I}\right)_{E}\right\| \|_{E}^{2} \\
& =\left.4 \mu_{E}^{2}\left\|\left(\left(\nabla \mathbf{w}^{1}-\frac{1}{3} \operatorname{div} \mathbf{w}^{1} \mathbb{I}\right)_{I}\right)_{E}\right\|\right|_{E} ^{2}
\end{aligned}
$$

which, following the same interpolation-scaling argument adopted in (3.39)-(3.40), yields

$$
\left\|\boldsymbol{\delta}_{E}-\frac{1}{3} \operatorname{tr}_{E}\left(\boldsymbol{\delta}_{E}\right) \mathbb{I}_{E}\right\|_{E}^{2} \leq 4 \mu_{E}^{2}\left\|\nabla \mathbf{w}^{1}-\frac{1}{3} \operatorname{div} \mathbf{w}^{1} \mathbb{I}\right\|_{L^{2}(E)}^{2} \leq C \mu_{E}^{2}\left\|\nabla \mathbf{w}^{1}\right\|_{L^{2}(E)}^{2} .
$$

The result follows by combining bounds (4.18) and (4.21).

We are now able to prove property (2.25).

Proposition 4.1. It exists a positive constant $C_{*}$, independent of $h, \lambda, \mu$, such that

$$
\left[\boldsymbol{\tau}_{E}, \boldsymbol{\tau}_{E}\right]_{E} \geq \frac{C_{*}}{\mu_{E}}\|\| \boldsymbol{\tau}_{E}-\frac{1}{3} \operatorname{tr}_{E}\left(\boldsymbol{\tau}_{E}\right) \mathbb{I}_{E}\|\|_{E}^{2}
$$

for all $\tau_{h} \in X_{h}$. 
Proof. In view of the identification introduced at the beginning of this section, a scaling argument gives

$$
\sum_{e \in \partial E} \frac{1}{|e|} \int_{e}\left|\boldsymbol{\tau}_{E}^{e}(\boldsymbol{\xi})\right|^{2} \mathrm{~d} \boldsymbol{\xi} \simeq \sum_{e \in \partial E}\left(\left\|\hat{\boldsymbol{\tau}}_{e}\right\|^{2}+\left\|\widetilde{\boldsymbol{\tau}}_{e}^{1}\right\|^{2}+\left\|\widetilde{\boldsymbol{\tau}}_{e}^{2}\right\|^{2}\right)=\left\|\boldsymbol{\tau}_{E}\right\|^{2}
$$

for all $\tau_{E}$ in $\left.X_{h}\right|_{E}$, where the symbol $\simeq$ indicates equivalence up to a constant uniform in $h, \mu$ and $\lambda$. Due to (4.23) and (M2) it follows that

$$
\begin{aligned}
|E|\left\|\boldsymbol{\tau}_{E}\right\|^{2} & \simeq \sum_{e \in \partial E} \frac{|E|}{|e|} \int_{e}\left|\boldsymbol{\tau}_{E}^{e}(\boldsymbol{\xi})\right|^{2} \mathrm{~d} \boldsymbol{\xi} \\
& \simeq \sum_{e \in \partial E} h_{e} \int_{e}\left|\boldsymbol{\tau}_{E}^{e}(\boldsymbol{\xi})\right|^{2}=\left.||\left|\boldsymbol{\tau}_{E}\right|||_{E}^{2} \quad \forall \boldsymbol{\tau}_{E} \in X_{h}\right|_{E}
\end{aligned}
$$

Following the decomposition in (4.9), we set

$$
\boldsymbol{\tau}_{E}=\boldsymbol{\tau}_{1}+\boldsymbol{\tau}_{2}, \quad \boldsymbol{\tau}_{1} \in \operatorname{img}(\mathrm{N}), \boldsymbol{\tau}_{2} \in \operatorname{ker}(\mathrm{N})
$$

Recalling (4.3) and (4.10), by definition of $\mathrm{M}$ and $\mathrm{C}$ it follows that

$$
\left[\boldsymbol{\tau}_{E}, \boldsymbol{\tau}_{E}\right]_{E}=\boldsymbol{\tau}_{E}^{T} \mathrm{M}_{E} \boldsymbol{\tau}_{E}=\boldsymbol{\tau}_{1}^{T} \mathrm{M}_{1} \boldsymbol{\tau}_{1}+\boldsymbol{\tau}_{2}^{T} \mathrm{M}_{1} \boldsymbol{\tau}_{2}+2 \boldsymbol{\tau}_{1}^{T} \mathrm{M}_{1} \boldsymbol{\tau}_{2}+\boldsymbol{\tau}_{2}^{T} \mathrm{M}_{2} \boldsymbol{\tau}_{2}
$$

Observing that, by definition, M1 is symmetric and semi-positive definite, we have that

$$
2 \boldsymbol{\tau}_{1}^{T} \mathrm{M}_{1} \boldsymbol{\tau}_{2} \geq-2\left(\boldsymbol{\tau}_{1}^{T} \mathrm{M}_{1} \boldsymbol{\tau}_{1}\right)^{1 / 2}\left(\boldsymbol{\tau}_{2}^{T} \mathrm{M}_{1} \boldsymbol{\tau}_{2}\right)^{1 / 2} \geq-\delta \boldsymbol{\tau}_{1}^{T} \mathrm{M}_{1} \boldsymbol{\tau}_{1}-\frac{1}{\delta} \boldsymbol{\tau}_{2}^{T} \mathrm{M}_{1} \boldsymbol{\tau}_{2}
$$

where at the present level the real number $0<\delta<1$.

Due to the inclusion $\boldsymbol{\tau}_{2} \in \operatorname{ker}(\mathrm{N})$ and the definition of $\mathrm{C}$, we have that $\boldsymbol{\tau}_{2}=\mathrm{C}_{2}$ for some $\mathbf{v}_{2} \in \mathbb{R}^{9 n-9}$. Therefore, recalling the definition of $\mathrm{M}_{2}$ and that the columns of $\mathrm{C}$ are orthogonal unit vectors, some simple algebra gives

$$
\tau_{2}^{T} \mathrm{M}_{2} \boldsymbol{\tau}_{2}=\mathbf{v}_{2}^{T} \mathrm{C}^{T} \mathrm{CUC}^{T} \mathrm{C} \mathbf{v}_{2}=\mathbf{v}_{2}^{T} \mathrm{U} \mathbf{v}_{2}
$$

which, adopting for simplicity the choice (4.11) gives

$$
\boldsymbol{\tau}_{2}^{T} \mathrm{M}_{2} \boldsymbol{\tau}_{2}=\frac{|E|}{\mu_{E}}\left\|\mathbf{v}_{2}\right\|^{2}=\frac{|E|}{\mu_{E}}\left\|\boldsymbol{\tau}_{2}\right\|^{2}
$$

First, using bound (4.27) and Lemma 4.1, then applying (4.29), identity (4.26) yields

$$
\begin{aligned}
{\left[\boldsymbol{\tau}_{E}, \boldsymbol{\tau}_{E}\right]_{E} } & \geq(1-\delta) \boldsymbol{\tau}_{1}^{T} \mathrm{M}_{1} \boldsymbol{\tau}_{1}+\boldsymbol{\tau}_{2}^{T} \mathrm{M}_{2} \boldsymbol{\tau}_{2}+\left(1-\frac{1}{\delta}\right) \boldsymbol{\tau}_{2}^{T} \mathrm{M}_{1} \boldsymbol{\tau}_{2} \\
& \geq(1-\delta) \boldsymbol{\tau}_{1}^{T} \mathrm{M}_{1} \boldsymbol{\tau}_{1}+\boldsymbol{\tau}_{2}^{T} \mathrm{M}_{2} \boldsymbol{\tau}_{2}+C_{1} \frac{|E|}{\mu_{E}}\left(1-\frac{1}{\delta}\right)\left\|\boldsymbol{\tau}_{2}\right\|^{2} \\
& =(1-\delta) \boldsymbol{\tau}_{1}^{T} \mathrm{M}_{1} \boldsymbol{\tau}_{1}+\frac{|E|}{\mu_{E}}\left(1+C_{1}\left(1-\frac{1}{\delta}\right)\right)\left\|\boldsymbol{\tau}_{2}\right\|^{2}
\end{aligned}
$$

Recalling that $\boldsymbol{\tau}_{1} \in \operatorname{img}(\mathrm{N})$ and using Lemma 4.2, yields

$$
(1-\delta) \boldsymbol{\tau}_{1}^{T} \mathrm{M}_{1} \boldsymbol{\tau}_{1} \geq \frac{C_{+}}{\mu_{E}}(1-\delta)\left|\left\|\boldsymbol{\tau}_{1}-\frac{1}{3} \operatorname{tr}_{E}\left(\boldsymbol{\tau}_{1}\right) \mathbb{I}_{E}\right\|\right|_{E}^{2}
$$


while equivalence (4.24) yields

$$
|E||| \boldsymbol{\tau}_{2} \|^{2} \geq\left. C_{3}|| \boldsymbol{\tau}_{2}||\right|_{E} ^{2}
$$

with the constant $C_{3}$ independent of $h, \mu, \lambda$.

Using a triangle inequality, a scaling argument and definition (4.13), leads to

$$
\left.\left\|\boldsymbol{\tau}_{2}-\frac{1}{3} \operatorname{tr}_{E}\left(\boldsymbol{\tau}_{2}\right) \mathbb{I}_{E}\right\|\right|_{E} ^{2} \leq 2\left|\left\|\boldsymbol{\tau}_{2}\right\|\right|_{E}^{2}+2\left|\left\|\frac{1}{3} \operatorname{tr}_{E}\left(\boldsymbol{\tau}_{2}\right) \mathbb{I}_{E}\right\|\right|_{E}^{2} \leq\left. C_{4}\left\|\left|\boldsymbol{\tau}_{2}\right|\right\|\right|_{E} ^{2}
$$

where the positive constant $C_{4}$ is independent of $h, \mu, \lambda$.

By combining all the bounds (4.30)-(4.33) and choosing, for instance, $\delta=\frac{2 C_{1}}{1+2 C_{1}}$, we obtain the inequalities

$$
\begin{aligned}
{\left[\boldsymbol{\tau}_{E}, \boldsymbol{\tau}_{E}\right]_{E} \geq } & \frac{C_{+}}{\mu_{E}}(1-\delta) \mid\left\|\boldsymbol{\tau}_{1}-\frac{1}{3} \operatorname{tr}_{E}\left(\boldsymbol{\tau}_{1}\right) \mathbb{I}_{E}\right\| \|_{E}^{2} \\
& +\frac{C_{3} C_{4}^{-1}}{\mu_{E}}\left(1+C_{1}\left(1-\frac{1}{\delta}\right)\right)\|\| \boldsymbol{\tau}_{2}-\frac{1}{3} \operatorname{tr}_{E}\left(\boldsymbol{\tau}_{2}\right) \mathbb{I}_{E} \|\left.\right|_{E} ^{2} \\
\geq & \frac{C_{5}}{\mu_{E}} \sum_{i=1,2}\|\| \boldsymbol{\tau}_{i}-\frac{1}{3} \operatorname{tr}_{E}\left(\boldsymbol{\tau}_{i}\right) \mathbb{I}_{E} \|\left.\right|_{E} ^{2}
\end{aligned}
$$

where the positive constant

$$
C_{5}=\min \left\{\frac{C_{+}}{1+2 C_{1}}, \frac{C_{3} C_{4}^{-1}}{2}\right\} .
$$

The result follows from (4.34) and (4.25) with a triangle inequality.

In Lemma 4.2 and Proposition 4.1 we do not need to assume that $\operatorname{div}_{h} \boldsymbol{\tau}_{E}=0$ as it appears in (S2), because the constant functions $\mathbb{C} \nabla p_{j}^{1}$ appearing in the definition of $i m g(\mathrm{~N})$ automatically satisfy this condition. Nevertheless, we prefer to keep (S2) as it has been stated since this allows us to consider a more general array of scalar products, see also the remark below.

Remark 4.1. Another viable choice for the construction of the scalar product would be to follow the same steps but substituting the space $\left[\mathcal{P}_{1}(E)\right]^{3}$ in $(4.4)-(4.6)$ with the richer space $\left[\mathcal{P}_{2}(E)\right]^{3}$, in accordance with [8]. In such a way we would obtain a method that satisfies consistency (S2) up to second order polynomials. Such stronger consistency would lead to a more complex construction, while keeping the same order of convergence for the method. Nevertheless, it would be interesting to make a numerical comparison between the original and the " $\mathcal{P}_{2}$-consistent" scheme, in order to assess if such an additional computational effort might lead to a significant reduction of the error constant.

Remark 4.2. A mimetic scheme for the Stokes problem has been recently developed in [10]. The method of [10] can be trivially extended to (almost-incompressible) elasticity, yielding an MFD scheme that follows a displacement-pressure formulation instead of a stress-displacement one.

\section{REFERENCES}

[1] S. Agmon, Lectures on Elliptic Boundary Value Problems. Van Nostrand, USA (1965).

[2] M. Amara and J.M. Thomas, Equilibrium finite elements for the linear elastic problem. Numer. Math. 33 (1979) $367-383$.

[3] D.N. Arnold, F. Brezzi and J. Douglas Jr., PEERS: A new mixed finite element for plane elasticity. Japan J. Appl. Math. 1 (1984) 347-367.

[4] D.N. Arnold, R.S. Falk and R. Winther, Differential complexes and stability of finite element methods II: the elasticity complex, in Compatible Spatial Discretizations, D. Arnold, P. Botchev, R. Lehoucq, R. Nicolaides and M. Shashkov Eds., IMA Volumes in Mathematics and its Applications 142, Springer-Verlag (2005) 47-67.

[5] D.N. Arnold, R.S. Falk and R. Winther, Mixed finite element methods for linear elasticity with weakly imposed symmetry. Math. Comp. 76 (2007) 1699-1723. 
[6] L. Beirão da Veiga, A residual based error estimator for the Mimetic Finite Difference method. Numer. Math. 108 (2008) 387-406.

[7] L. Beirão da Veiga and G. Manzini, An a posteriori error estimator for the mimetic finite difference approximation of elliptic problems with general diffusion tensors. Int. J. Num. Meth. Engrg. 76 (2008) 1696-1723.

[8] L. Beirão da Veiga and G. Manzini, A higher-order formulation of the Mimetic Finite Difference method. SIAM J. Sci. Comput. 31 (2008) 732-760.

[9] L. Beirão da Veiga, K. Lipnikov and G. Manzini, Convergence analysis of the high-order mimetic finite difference method. Numer. Math. 113 (2009) 325-356.

[10] L. Beirão da Veiga, V. Gyrya, K. Lipnikov and G. Manzini, A mimetic finite difference method for the Stokes problem on polygonal meshes. J. Comput. Phys. 228 (2009) 7215-7232.

[11] M. Berndt, K. Lipnikov, J.D. Moulton and M. Shashkov, Convergence of mimetic finite difference discretizations of the diffusion equation. J. Numer. Math. 9 (2001) 253-284.

[12] M. Berndt, K. Lipnikov, M. Shashkov, M.F. Wheeler and I. Yotov, Superconvergence of the velocity in mimetic finite difference methods on quadrilaterals. SIAM J. Numer. Anal. 43 (2005) 1728-1749.

[13] F. Brezzi and M. Fortin, Mixed and Hybrid Finite Element Methods. Springer-Verlag, New York, USA (1991).

[14] F. Brezzi, J. Douglas Jr. and L.D. Marini, Two families of mixed finite elements for second order elliptic problems. Numer. Math. 47 (1985) 217-235.

[15] F. Brezzi, D. Boffi and M. Fortin, Reduced symmetry elements in linear elasticity. Comm. Pure Appl. Anal. 8 (2009) 95-121.

[16] F. Brezzi, K. Lipnikov and M. Shashkov, Convergence of mimetic finite difference method for diffusion problems on polyhedral meshes. SIAM J. Numer. Anal. 43 (2005) 1872-1896.

[17] F. Brezzi, K. Lipnikov and V. Simoncini, A family of mimetic finite difference methods on polygonal and polyhedral meshes. Math. Models Methods Appl. Sci. 15 (2005) 1533-1553.

[18] F. Brezzi, K. Lipnikov and V. Simoncini, Convergence of mimetic finite difference methods for diffusion problems on polyhedral meshes with curved faces. Math. Models Methods Appl. Sci. 16 (2006) 275-298.

[19] F. Brezzi, K. Lipnikov, M. Shashkov and V. Simoncini, A new discretization methodology for diffusion problems on generalized polyhedral meshes. Comp. Meth. Appl. Mech. Engrg. 196 (2007) 3682-3692.

[20] A. Cangiani and G. Manzini, Flux recontruction and pressure post-processing in mimetic finite difference methods. Comput. Meth. Appl. Mech. Engrg. 197 (2008) 933-945.

[21] P.G. Ciarlet, Mathematical Elasticity, Volume I: Three-Dimensional Elasticity, Studies in Mathematics and its Applications 20. Amsterdam, North Holland (1988).

[22] B.X. Fraejis de Vebeuke, Stress function approach, in World Congress on the Finite Element Method in Structural Mechanics, Bornemouth (1975).

[23] V. Gryrya and K. Lipnikov, High-order mimetic finite difference method for the diffusion problems on polygonal meshes. J. Comput. Phys. 227 (2008) 8841-8854.

[24] J. Hyman, M. Shashkov and S. Steinberg, The numerical solution of diffusion problems in strongly heterogeneus non-isotropic materials. J. Comput. Phys. 132 (1997) 130-148.

[25] J. Hyman, J. Morel, M. Shashkov and S. Steinberg, Mimetic finite difference methods for diffusion equations. Comput. Geosci. 6 (2002) 333-352.

[26] Y. Kuznetsov, K. Lipnikov and M. Shashkov, The mimetic finite difference method on polygonal meshes for diffusion-type problems. Comput. Geosci. 8 (2005) 301-324.

[27] K. Lipnikov, J. Morel and M. Shashkov, Mimetic finite difference methods for diffusion equations on non-orthogonal nonconformal meshes. J. Comput. Phys. 199 (2004) 589-597.

[28] K. Lipnikov, M. Shashkov and D. Svyatskiy, The mimetic finite difference discretization of diffusion problem on unstructured polyhedral meshes. J. Comput. Phys. 211 (2006) 473-491.

[29] J. Morel, M. Hall and M. Shaskov, A local support-operators diffusion discretization scheme for hexahedral meshes. J. Comput. Phys. 170 (2001) 338-372. 